\title{
Approximations to Limit Cycles for a Nonlinear Multi-Degree-of-Freedom System with a Cubic Nonlinearity through Combining the Harmonic Balance Method with Perturbation Techniques
}

\author{
A. P. Lewis \\ School of Engineering and Computer Science, University of Hertfordshire, Hatfield, Hertfordshire \\ AL10 9AB, United Kingdom. E-mail: a.lewis@ herts.ac.uk
}

\begin{abstract}
This paper presents an approach to obtaining higher order approximations to limit cycles of an autonomous multi-degree-of-freedom system with a single cubic nonlinearity based on a first approximation involving first and third harmonics obtained with the harmonic balance method. This first approximation, which is similar to one which has previously been reported in the literature, is an analytical solution, except that the frequency has to be obtained numerically from a polynomial equation of degree 16. An improved solution is then obtained in a perturbation procedure based on the refinement of the harmonic balance solution. The stability of the limit cycles obtained is then investigated using Floquet analysis.

The capability of this approach to refine the results obtained by the harmonic balance first approximation is demonstrated, by direct comparison with time domain simulation and frequency components obtained using the Discrete Fourier Transform. The particular case considered was based on an aeroelastic analysis of an all-moving control surface with a nonlinearity in the torsional degreeof-freedom of the root support, and parameters corresponding to air speed, together with linear stiffness and viscous damping of the root support were varied. It is also shown, for the cases considered, how the method can reveal further bifurcational behaviour of the system beyond the initial Hopf bifurcations which first lead to the onset of limit cycle oscillations.
\end{abstract}

KEYWORDS: Limit cycle oscillations; harmonic balance, perturbation methods, Floquet Analysis

This research did not receive any specific grant from funding agencies in the public, commercial, or not-for-profit sectors.

\section{Introduction}

Methods for the analysis of nonlinear autonomous systems of ordinary differential equations have been the subject of considerable investigation over many years, from both a purely theoretical viewpoint and also in the context of practical applications. Nonlinear ordinary differential equations may, of course, be solved numerically in the time domain. However, a drawback with this is that though it can yield a complete picture of system behaviour for a given set of initial conditions, it can be inefficient in providing an overall picture of system characteristics. Besides this, for nonlinear autonomous systems that arise in practical applications, it is often necessary to solve them for a wide range of system parameters. Analysis based on averaging or other methods of asymptotic analysis has the advantage of being able to yield both qualitative, and in many cases the most important quantitative, information about the system response relatively rapidly, thus enabling an understanding of the system's behaviour to be obtained more quickly. Of particular interest is the determination of limit cycles and their stability for autonomous systems. The literature on analytical methods for solving nonlinear systems is extensive and includes classical perturbation methods such as the Krylov-Bogoliubov, Krylov-BogoliubovMitropolsky methods and generalised averaging [1,2]. Equally, considerable research into analytical methods applicable in the case of strong nonlinearities, where perturbation methods may cease to be applicable, has been carried out and a broad coverage of such approaches are presented in [3], for 
example. A wide range of second order, single-degree-of-freedom systems in particular have been tackled, both conservative and dissipative. For multi-degree-of-freedom systems possessing strong nonlinearities, there is a very large body of literature on wholly numerically-based approaches based on the harmonic balance method, many concerned with development of algorithms, for example [4 - 9] while many are also concerned with applications, in particular in the field of nonlinear aeroelasticity [10 - 16]. Methodologies which combine analytical and numerical techniques have also been developed and include perturbation-incremental and perturbation-iterative methods [17 - 20]. Other methods that have been developed are the differential transform method [21], He's homotopy method [22] and an integro-differential equation approach [23]. A further approach, applicable to systems with a cubic nonlinearity, makes use of Jacobi elliptic functions. A review of this method, with applications to single degree-of-freedom second order systems, has been carried out by Kovacic et al. [24]. The method, in the form of elliptic harmonic balance, has been applied to aeroelastic systems possessing cubic nonlinearities $[25,26]$. There has also been interest in the application of the Groebner basis method to solving the coupled polynomial equations which may arise from the harmonic balance method $[27,28]$. As the Groebner basis approach leads ultimately to the solution of polynomial equations, the potential advantage of this approach is in being better able to capture possible periodic solutions. Peddieson et Liu [27], for example, analyse a second order single degree-of-freedom system with sinusoidal forcing, and free undamped vibration of a second order single degree-of-system system [28]. A potential drawback, however, is in the practicalities of how many harmonics can be included. Grolet et al [29] combine use of the Groebner basis method initially for an undamped system with continuation on a damping parameter to obtain periodic solutions for a damped system. Lee et al [30] obtain a harmonic balance solution, using first and third harmonics, to a nonlinear aeroelastic problem involving a pitching and plunging aerofoil in incompressible flow. They derive a polynomial equation for frequency of oscillation without the use of Groebner bases after which the coefficients in the harmonic balance formulation are readily obtained.

In this paper, limit cycle oscillations for a coupled second order system with a single cubic nonlinearity are investigated. The starting point of the approach taken is the analysis of Lee et al. [30] who, as already mentioned, obtained a harmonic balance solution, using first and third harmonics, to a second order multi-degree-of-freedom system. The solution can be thought of as almost analytical in the sense that a polynomial equation for frequency of oscillation is obtained, but this equation is of high degree and does need to be solved numerically. Other than this, the solution would be wholly analytical. In this way, a first approximation is thus obtained. To incorporate the effect of higher harmonics an approach similar to that adopted by Qui and Filanovsky [31] is taken, who obtained approximations to limit cycle oscillations of the Van der Pol equation for moderate values of damping coefficient. The basis of their method was to start with an approximate solution obtained by harmonic balance which they then refined. A further application of this approach is presented in [32]. It might be noted at this point that in [30], refinements to harmonic balance solutions based on the first harmonic alone were also obtained using Popov's method [33]. Having obtained approximations to the limit cycles of the nonlinear system, Floquet analysis is applied to investigate their stability. The approach taken is that of [23] and involved extending to a two degree-of-freedom second order, or equivalently, a four degree-of-freedom first order system, a method applied by Bonani [34] to Chua's circuit, which is a three degree-of-freedom system. The contribution made by this paper is therefore through extending the method of [31] to a multi-degree-of-freedom system and basing it on an available harmonic balance solution containing not only first, but also third harmonics.

The particular case studied in this paper is a two degree-of-freedom second order system with a cubic nonlinearity motivated by, and based on, an aeroelastic analysis of an all-moving control surface in supersonic flow where piston theory aerodynamics [35] may be applied. The layout of the paper is as follows: Section 2 presents the first approximation obtained from the harmonic balance solution involving first and third harmonics in a manner similar to that of [30]. Section 3 presents the refined solution obtained with a perturbation solution based on the approach of [31]. Section 4 outlines the general approach adopted for analysing the stability of any limit cycle solutions obtained. Sections 5 presents results and Section 6 Concluding Remarks. 


\section{Initial Harmonic Balance Solution}

In this study, the following autonomous nonlinear system is considered:

$$
\begin{gathered}
\ddot{X}_{1}+G_{11} \dot{X}_{1}+G_{12} \dot{X}_{2}+H_{11} X_{1}+H_{12} X_{2}+\mu X_{1}^{3}=0 \\
\ddot{X}_{2}+G_{21} \dot{X}_{1}+G_{22} \dot{X}_{2}+H_{21} X_{1}+H_{22} X_{2}=0
\end{gathered}
$$

where $X_{1}, X_{2}$ are the dependent variables. $G_{\mathrm{ij}}$ and $H_{\mathrm{ij}}$ (where $\mathrm{i}, \mathrm{j}=1,2$ ) are damping and stiffness coefficients resulting in damping and stiffness matrices which are not necessarily symmetric, as might be the case in aeroelastic applications, for example. $\mu$ governs the strength of the nonlinearity. Derivatives are with respect to time $t$. In this section, a harmonic balance approach to obtain limit cycle solutions, incorporating first and third harmonics, is first presented, via an approach based on that of Lee et al. [30], and which will result in a polynomial equation for the fundamental frequencies of oscillation of any limit cycles. Given these frequencies, the limit cycles are then determined analytically. Hence, the only numerical part of this approach is in solving a polynomial equation for frequency. It should be noted that in [30], an aeroelastic problem involving incompressible flow is tackled in which the aerodynamics are characterised via a state-space approach, resulting in a more complex situation than that considered here.

The first step in the analysis is to introduce a non-dimensional time $\tau$ where $\tau=\omega t, \omega$ being the fundamental frequency of the periodic solution of (1) being sought. Equation (1) is now rewritten:

$$
\begin{gathered}
\omega^{2} X_{1}^{\prime \prime}+\omega G_{11} X_{1}^{\prime}+\omega G_{12} X_{2}^{\prime}+H_{11} X_{1}+H_{12} X_{2}+\mu X_{1}^{3}=0 \\
\omega^{2} X_{2}^{\prime \prime}+\omega G_{21} X_{1}^{\prime}+\omega G_{22} X_{2}^{\prime}+H_{21} X_{1}+H_{22} X_{2}=0
\end{gathered}
$$

where ' denotes differentiation with respect to $\tau$.

Following [30], a periodic solution is now obtained of the form:

$$
\begin{gathered}
X_{1}=a_{1} \sin \tau+a_{3} \sin 3 \tau+b_{3} \cos 3 \tau \\
X_{2}=c_{1} \sin \tau+d_{1} \cos \tau+c_{3} \sin 3 \tau+d_{3} \cos 3 \tau
\end{gathered}
$$

where one is free not to have a $\cos \tau$ term in the first of Equations (3) as an autonomous system is being considered. The choice of harmonics in Equation (3) will enable significant nonlinear effects to be investigated. The possible extension to solving analytically the equations which would arise if even, together with higher odd harmonics, were included would involve significant extra complexity but is a good area for further investigation. Substituting Equations (3) into (2) and equating coefficients of $\cos \tau$, $\sin \tau, \cos 3 \tau, \sin 3 \tau$ leads to the following:

$$
\begin{gathered}
m_{1} a_{1}+p_{1} c_{1}+q_{1} d_{1}=0 \\
m_{2} a_{1}+p_{2} c_{1}+q_{2} d_{1}=0 \\
m_{13} a_{3}+n_{13} b_{3}+p_{13} c_{3}+q_{13} d_{3}=0 \\
m_{23} a_{3}+n_{23} b_{3}+p_{23} c_{3}+q_{23} d_{3}=0
\end{gathered}
$$




$$
\begin{gathered}
m_{3} a_{1}+p_{3} c_{1}+q_{3} d_{1}-\frac{3}{4} \mu a_{1}^{2} b_{3}=0 \\
m_{4} a_{1}+p_{4} c_{1}+q_{4} d_{1}+\mu\left\{\frac{3}{4} a_{1}^{3}-\frac{3}{4} a_{1}^{2} a_{3}+\frac{3}{2} a_{1}\left(a_{3}^{2}+b_{3}^{2}\right)\right\}=0 \\
m_{33} a_{3}+n_{33} b_{3}+p_{33} c_{3}+q_{33} d_{3}+\mu\left\{\frac{3}{2} a_{1}^{2} b_{3}+\frac{3}{4} b_{3}\left(a_{3}^{2}+b_{3}^{2}\right)\right\}=0 \\
m_{43} a_{3}+n_{43} b_{3}+p_{43} c_{3}+q_{43} d_{3}+\mu\left\{-\frac{1}{4} a_{1}^{3}+\frac{3}{2} a_{1}^{2} a_{3}+\frac{3}{2} a_{3}\left(a_{3}^{2}+b_{3}^{2}\right)\right\}=0
\end{gathered}
$$

The coefficients of $a_{1}, a_{3}, b_{3}, c_{1}, d_{1}, c_{3}, d_{3}$ in Equations (4) to (7) are functions of the coefficients of $G_{\mathrm{ij}}$ and $H_{\mathrm{ij}}$ together with $\omega$, and expressions for them are given in Appendix A.

Using Equations (4) and (5), $c_{1}, d_{1}, c_{3}, d_{3}$ may be expressed in terms $a_{1}, a_{3}, b_{3}$ and eliminated from Equations (6) and (7) leading to the following:

$$
\begin{gathered}
M_{3} a_{1}-\frac{3}{4} \mu R_{1}^{2} b_{3}=0 \\
M_{4} a_{1}+\mu\left\{\frac{3}{4} R_{1}^{2} a_{1}-\frac{3}{4} R_{1}^{2} a_{3}+\frac{3}{2} a_{1} R_{3}^{2}\right\}=0 \\
M_{33} a_{3}+N_{33} b_{3}+\mu\left\{\frac{3}{2} R_{1}^{2} b_{3}+\frac{3}{4} b_{3} R_{3}^{2}\right\}=0 \\
M_{43} a_{3}+N_{43} b_{3}+\mu\left\{-\frac{1}{4} R_{1}^{2} a_{1}+\frac{3}{2} R_{1}^{2} a_{3}+\frac{3}{4} a_{3} R_{3}^{2}\right\}=0
\end{gathered}
$$

where $R_{1}^{2}=a_{1}^{2}, R_{3}^{2}=a_{3}^{2}+b_{3}^{2}$ and the coefficients of $a_{1}, a_{3}, b_{3}$ are given in Appendix A.

As indicated in Appendix A, $M_{33}=-N_{43}$ and $M_{43}=N_{33}$. Hence, from Equations (8), (10) and (11), it is possible to obtain the relationship:

$$
3 N_{43} R_{3}^{2}=M_{3} R_{1}^{2}
$$

while from Equations (9), (10) and (11), the following equation may be obtained for $R_{1}^{2}$ :

$$
R_{1}^{2}=\frac{4 M_{33}\left(M_{3} M_{43}+M_{4} M_{33}\right)}{\mu\left(M_{3}^{2}-4 M_{3} M_{33}-3 M_{33}^{2}\right)}
$$

and hence using (12):

$$
R_{3}^{2}=-\frac{4 M_{3}\left(M_{3} M_{43}+M_{4} M_{33}\right)}{3 \mu\left(M_{3}^{2}-4 M_{3} M_{33}-3 M_{33}^{2}\right)}
$$


Equations (13) and (14) are actually the same as Equations (28) and (29) in [30] where $M_{3}$ and $M_{33}$ correspond to $M_{4}$ and $M_{43}$ in this paper and vice-versa. From Equations (13) and (14), $R_{1}^{2}$ and $R_{3}^{2}$ are now functions of $\omega$ only. Equations (8) to (11) may now be solved for $a_{1}, a_{3}, b_{3}$ and $\omega$. From (9), it is possible to obtain the following expression for $a_{3}$ :

$$
a_{3}=\frac{4}{3 \mu R_{1}}\left\{M_{4}+\frac{3}{4} \mu R_{1}^{2}+\frac{3}{2} \mu R_{3}^{2}\right\}
$$

while from (8) it is possible to obtain the following expression for $b_{3}$ :

$$
b_{3}=\frac{4 M_{3}}{3 \mu R_{1}}
$$

Using the definition $R_{3}^{2}=a_{3}^{2}+b_{3}^{2}$ together with Equations (13) to (16) then leads to the following Equation for the frequency $\omega$ :

$$
\begin{gathered}
M_{3}^{5}-8 M_{33} M_{3}^{4}+\left(M_{4}^{2}-4 M_{4} M_{43}+10 M_{33}^{2}+4 M_{43}^{2}\right) M_{3}^{3}+ \\
\left(-12 M_{4}^{2} M_{33}+30 M_{4} M_{33} M_{43}+24 M_{33}^{3}-9 M_{33} M_{43}^{2}\right) M_{3}^{2}+ \\
\left(36 M_{4}^{2} M_{33}^{2}-30 M_{4} M_{33}^{2} M_{43}+9 M_{33}^{4}+9 M_{33}^{2} M_{43}^{2}\right) M_{3}+3 M_{4}^{2} M_{33}^{3}=0
\end{gathered}
$$

Making use of the expressions in Appendix A for $M_{3}, M_{4}, M_{33}, M_{43}$ then results in a polynomial equation for $\omega$. Both equation (17) and the explicit form of the polynomial equation are most readily obtained using a symbolic mathematics package. The resulting polynomial equation is of degree 35 in $\omega$. However the polynomial includes a factor $\omega^{3}$ so that removing this and setting $x=\omega^{2}$ leaves a polynomial of degree 16 in $\omega^{2}$, in contrast to the polynomial obtained in [30], which was of degree 37 in $\omega^{2}$ including zero roots (as a result of the state space representation of the incompressible unsteady aerodynamics). Once the polynomial equation in $x=\omega^{2}$ has been solved, $R_{1}^{2}$ and $R_{3}^{2}$ may be evaluated from (14) and (15) and the amplitudes $a_{1}, a_{3}, b_{3}, c_{1}, d_{1}, c_{3}, d_{3}$ obtained. For admissible solutions, it is required that $x=\omega^{2}, R_{1}^{2}$ and $R_{3}^{2}$ are all positive. As coefficients in Equations (2) are varied, none, one, two or possibly more periodic solutions may be obtained.

\section{Refined Solution}

The solution presented in Section 2 incorporates the effect of first and third harmonics. More accurate solutions will need to incorporate the effect of higher harmonics, and in this section, this is achieved by using perturbation techniques to build on the solution of Section 2 in an approach similar to that adopted in [31] for obtaining periodic solutions to the Van der Pol Equation for moderate values of damping coefficients. In this approach, the solution to (2) is written as follows:

$$
\begin{aligned}
& X_{1}=X_{10}+\varepsilon X_{11}+\varepsilon^{2} X_{12}+\cdots \\
& X_{2}=X_{20}+\varepsilon X_{21}+\varepsilon^{2} X_{22}+\cdots
\end{aligned}
$$

with the frequency of oscillation, $\omega$, written as:

$$
\omega=\omega_{0}+\varepsilon \omega_{1}+\varepsilon^{2} \omega_{2}+\cdots
$$

The terms $X_{10}, X_{20}, \omega_{0}$ in fact represent the solution already obtained in Section 2. The terms $X_{11}, X_{12}$, $X_{21}, X_{22}, \ldots \ldots, \omega_{1}, \omega_{2}, \ldots$ represent small correction terms. $\varepsilon$ is a term used here to indicate the smallness of the correction terms and will be used in what follows in a process of equating terms having the same 
order of magnitude. $\varepsilon$ will then take the value 1 in the final results. It is now possible to write the nonlinear term $X_{1}^{3}$ in the form:

$$
f\left(X_{1}\right)=X_{1}^{3}=F_{1}+\left(f_{0}-F_{1}\right)+\left(f-f_{0}\right)
$$

where the term $f_{0}=f_{0}\left(X_{10}\right)$ is a function of $X_{10}$ only and so can be expanded as a Fourier series whose coefficients are known and will comprise a part made up of first and third harmonics, which will be denoted by $F_{1}$ together with a remainder $\left(f_{0}-F_{1}\right)$ made up of fifth, seventh and ninth harmonics, which can be taken as small, and hence can be treated as $\mathrm{O}(\varepsilon)$. Similarly, $f-f_{0}$ may be treated as small and thus, following [31], Equation (20) may be rewritten:

$$
f=F_{1}+\varepsilon\left(f_{0}-F_{1}\right)+\varepsilon\left(f-f_{0}\right)
$$

to highlight these small terms. In other words, $\varepsilon$ is introduced as a means of flagging small terms. When $\varepsilon$ is set to 1, Equation (20) is recovered. Equations (2) may now be re-written:

$$
\begin{gathered}
\omega^{2} X_{1}^{\prime \prime}+\omega G_{11} X_{1}^{\prime}+\omega G_{12} X_{2}^{\prime}+H_{11} X_{1}+H_{12} X_{2}+\mu F_{1}+\mu \varepsilon\left(f_{0}-F_{1}\right)+\mu \varepsilon\left(f-f_{0}\right)=0 \\
\omega^{2} X_{2}^{\prime \prime}+\omega G_{21} X_{1}^{\prime}+\omega G_{22} X_{2}^{\prime}+H_{21} X_{1}+H_{22} X_{2}=0
\end{gathered}
$$

so that a perturbation analysis may now be carried out based on powers of $\varepsilon$. However, the function $f$ defined in Equation (12) is now distinct from the original cubic nonlinearity and hence, at this stage, a different problem from (1) is now being tackled. Once $\varepsilon$ is set to 1 , it is anticipated that the result obtained approximates the solution of (1). This general approach is referred to as Parameter Expansion in [3]. A number of examples are presented therein showing the effectiveness of the approach. Its Advantages are discussed, in particular the applicability of the method to Truly Nonlinear Systems, but also the issues in justifying the process a priori. Substituting (18) and (19) into $f\left(X_{I}\right)$ then leads to the following expression:

$$
\begin{aligned}
f\left(X_{1}\right) & =X_{1}^{3}=f_{0}\left(X_{10}\right)+\varepsilon f_{1}\left(X_{10}, X_{11}\right)+\varepsilon^{2} f_{2}\left(X_{10}, X_{11}, X_{12}\right)+\cdots \\
& =X_{10}^{3}+\varepsilon\left(3 X_{10}^{2} X_{11}\right)+\varepsilon^{2}\left(3 X_{10}^{2} X_{12}+3 X_{10} X_{11}^{2}\right)+\cdots
\end{aligned}
$$

Equations (18), (19) and (23) may then be substituted in (22) and equating powers of $\varepsilon$ will give the following:

For O(1) terms:

$$
\begin{gathered}
\omega_{0}^{2} X_{10}^{\prime \prime}+\omega_{0} G_{11} X_{10}^{\prime}+\omega_{0} G_{12} X_{20}^{\prime}+H_{11} X_{10}+H_{12} X_{20}+\mu F_{1}=0 \\
\omega_{0}^{2} X_{20}^{\prime \prime}+\omega_{0} G_{21} X_{10}^{\prime}+\omega_{0} G_{22} X_{20}^{\prime}+H_{21} X_{10}+H_{22} X_{20}=0
\end{gathered}
$$

For $\mathrm{O}(\varepsilon)$ terms:

$$
\begin{gathered}
\omega_{0}^{2} X_{11}^{\prime \prime}+2 \omega_{0} \omega_{1} X_{10}^{\prime \prime}+\omega_{1} G_{11} X_{10}^{\prime}+\omega_{1} G_{12} X_{20}^{\prime}+\omega_{0} G_{11} X_{11}^{\prime}+\omega_{0} G_{12} X_{21}^{\prime}+H_{11} X_{11} \\
+H_{12} X_{21}+\mu\left(f_{0}-F_{1}\right)=0 \\
\omega_{0}^{2} X_{21}^{\prime \prime}+2 \omega_{0} \omega_{1} X_{20}^{\prime \prime}+\omega_{1} G_{21} X_{10}^{\prime}+\omega_{1} G_{22} X_{20}^{\prime}+\omega_{0} G_{21} X_{11}^{\prime}+\omega_{0} G_{22} X_{21}^{\prime}+H_{21} X_{11} \\
+H_{22} X_{21}=0
\end{gathered}
$$


For $\mathrm{O}\left(\varepsilon^{2}\right)$ terms:

$$
\begin{aligned}
\omega_{0}^{2} X_{12}^{\prime \prime} & +2 \omega_{0} \omega_{1} X_{11}^{\prime \prime}+\left(\omega_{1}^{2}+2 \omega_{0} \omega_{2}\right) X_{10}^{\prime \prime}+G_{11}\left(\omega_{0} X_{12}^{\prime}+\omega_{1} X_{11}^{\prime}+\omega_{2} X_{10}^{\prime}\right) \\
& +G_{12}\left(\omega_{0} X_{22}^{\prime}+\omega_{1} X_{21}^{\prime}+\omega_{2} X_{20}^{\prime}\right)+H_{11} X_{12}+H_{12} X_{22}+3 \mu X_{10}^{2} X_{11}=0 \\
\omega_{0}^{2} X_{22}^{\prime \prime} & +2 \omega_{0} \omega_{1} X_{21}^{\prime \prime}+\left(\omega_{1}^{2}+2 \omega_{0} \omega_{2}\right) X_{20}^{\prime \prime}+G_{21}\left(\omega_{0} X_{12}^{\prime}+\omega_{1} X_{11}^{\prime}+\omega_{2} X_{10}^{\prime}\right) \\
& +G_{22}\left(\omega_{0} X_{22}^{\prime}+\omega_{1} X_{21}^{\prime}+\omega_{2} X_{20}^{\prime}\right)+H_{21} X_{12}+H_{22} X_{22}=0
\end{aligned}
$$

Noting that in Equations (24), $F_{1}$ consists only of first and third harmonic terms, $X_{10}, X_{20}$ will be taken as:

$$
\begin{gathered}
X_{10}=a_{10} \sin \tau+a_{30} \sin 3 \tau+b_{30} \cos 3 \tau \\
X_{20}=c_{10} \sin \tau+d_{10} \cos \tau+c_{30} \sin 3 \tau+d_{30} \cos 3 \tau
\end{gathered}
$$

Substituting into Equations (24) and using harmonic balance to determine $a_{10}, a_{30}, b_{30}, c_{10}, d_{10}, c_{30}, d_{30}$, together with $\omega_{0}$ leads to the solution already obtained in Section 2 .

$f_{0}\left(X_{10}\right)$ and $F_{1}$ may now be written respectively as:

$$
\begin{gathered}
f_{0}\left(X_{10}\right)=C_{10} \cos \tau+S_{10} \sin \tau+C_{30} \cos 3 \tau+S_{30} \sin 3 \tau+C_{50} \cos 5 \tau+S_{50} \sin 5 \tau+ \\
C_{70} \cos 7 \tau+S_{70} \sin 7 \tau+C_{90} \cos 9 \tau+S_{90} \sin 9 \tau \\
F_{1}=C_{10} \cos \tau+S_{10} \sin \tau+C_{30} \cos 3 \tau+S_{30} \sin 3 \tau
\end{gathered}
$$

where $C_{10}, S_{10}, C_{30}, S_{30}, C_{50}, S_{50}, C_{70}, S_{70}, C_{90}, S_{90}$ are given in Appendix B and will be known in terms of $a_{10}, a_{30}, b_{30}, c_{10}, d_{10}, c_{30}, d_{30}$.

In the $\mathrm{O}(\varepsilon)$ Equation (25), in view of Equation (27), $X_{11}$, and $X_{21}$ are taken in the form:

$$
\begin{aligned}
X_{11}= & a_{11} \sin \tau+a_{31} \sin 3 \tau+b_{31} \cos 3 \tau+a_{51} \sin 5 \tau+b_{51} \cos 5 \tau+ \\
& a_{71} \sin 7 \tau+b_{71} \cos 7 \tau+a_{91} \sin 9 \tau+b_{91} \cos 9 \tau \\
X_{21}= & c_{11} \sin \tau+d_{11} \cos \tau+c_{31} \sin 3 \tau+d_{31} \cos 3 \tau+c_{51} \sin 5 \tau+ \\
& d_{51} \cos 5 \tau+c_{71} \sin 7 \tau+d_{71} \cos 7 \tau+c_{91} \sin 9 \tau+d_{91} \cos 9 \tau
\end{aligned}
$$

Note that in the expression for $X_{11}$, there is no $\cos \tau$ term to maintain consistency with the first approximation. Equations (27) - (29) are then substituted into (25) and harmonic balance is then carried out, leading to a set of linear equations for $a_{11}, a_{31}, b_{31}, a_{51}, b_{51}, a_{71}, b_{71}, a_{91}, b_{91}, c_{11}, d_{11}, c_{31}, d_{31}, c_{51}, d_{51}$, $c_{71}, d_{71}, c_{91}, d_{91}$, and $\omega_{1}$. In doing so, it emerges that since $f_{0}-F_{1}$ only includes $5^{\text {th }}$ harmonics and higher, it follows that $a_{11}, a_{31}, b_{31}, c_{11}, d_{11}, c_{31}, d_{31}$, and $\omega_{1}$ will all be zero. Hence the $\mathrm{O}(\varepsilon)$ corrections do not modify the frequency of oscillation or the first and third harmonics. The following equations are then obtained for the remaining coefficients:

$$
\begin{gathered}
\left(H_{11}-25 \omega_{0}^{2}\right) a_{51}-5 \omega_{0} G_{11} b_{51}+H_{12} c_{51}-5 \omega_{0} G_{12} d_{51}+\mu S_{50}=0 \\
5 \omega_{0} G_{11} a_{51}+\left(H_{11}-25 \omega_{0}^{2}\right) b_{51}+5 \omega_{0} G_{12} c_{51}+H_{12} d_{51}+\mu C_{50}=0 \\
H_{21} a_{51}-5 \omega_{0} G_{21} b_{51}+\left(H_{22}-25 \omega_{0}^{2}\right) c_{51}-5 \omega_{0} G_{22} d_{51}=0 \\
5 \omega_{0} G_{21} a_{51}+H_{21} b_{51}+5 \omega_{0} G_{22} c_{51}+\left(H_{22}-25 \omega_{0}^{2}\right) d_{51}=0
\end{gathered}
$$




$$
\begin{gathered}
\left(H_{11}-49 \omega_{0}^{2}\right) a_{71}-7 G_{11} \omega_{0} b_{71}+H_{12} c_{71}-7 \omega_{0} G_{12} d_{71}+\mu S_{70}=0 \\
7 \omega_{0} G_{11} a_{71}+\left(H_{11}-49 \omega_{0}^{2}\right) b_{71}+7 \omega_{0} G_{12} c_{71}+H_{12} d_{71}+\mu C_{70}=0 \\
H_{21} a_{71}-7 \omega_{0} G_{21} b_{71}+\left(H_{22}-49 \omega_{0}^{2}\right) c_{71}-7 \omega_{0} G_{22} d_{71}=0 \\
7 \omega_{0} G_{21} a_{71}+H_{21} b_{71}+7 \omega_{0} G_{22} c_{71}+\left(H_{22}-49 \omega_{0}^{2}\right) d_{71}=0 \\
\left(H_{11}-81 \omega_{0}^{2}\right) a_{91}-9 \omega_{0} G_{11} b_{91}+H_{12} c_{91}-9 \omega_{0} G_{12} d_{91}+\mu S_{90}=0 \\
9 \omega_{0} G_{11} a_{91}+\left(H_{11}-81 \omega_{0}^{2}\right) b_{91}+9 \omega_{0} G_{12} c_{91}+H_{12} d_{91}+\mu C_{90}=0 \\
H_{21} a_{91}-9 \omega_{0} G_{21} b_{91}+\left(H_{22}-81 \omega_{0}^{2}\right) c_{91}-9 \omega_{0} G_{22} d_{91}=0 \\
9 \omega_{0} G_{21} a_{91}+H_{21} b_{91}+9 \omega_{0} G_{22} c_{91}+\left(H_{22}-81 \omega_{0}^{2}\right) d_{91}=0
\end{gathered}
$$

Equations (30) to (32) are three separate sets of linear simultaneous equations which may be solved to give $a_{51}, b_{51}, a_{71}, b_{71}, a_{91}, b_{91}, c_{51}, d_{51}, c_{71}, d_{71}, c_{91}, d_{91}$.

In the $\mathrm{O}\left(\varepsilon^{2}\right)$ Equations (26) $X_{12}$, and $X_{22}$ are taken in the form:

$$
\begin{aligned}
X_{12}= & a_{12} \sin \tau+a_{32} \sin 3 \tau+b_{32} \cos 3 \tau+a_{52} \sin 5 \tau+b_{52} \cos 5 \tau+ \\
& a_{72} \sin 7 \tau+b_{72} \cos 7 \tau+a_{92} \sin 9 \tau+b_{92} \cos 9 \tau \\
X_{22}= & c_{12} \sin \tau+d_{12} \cos \tau+c_{32} \sin 3 \tau+d_{32} \cos 3 \tau+c_{52} \sin 5 \tau+ \\
& d_{52} \cos 5 \tau+c_{72} \sin 7 \tau+d_{72} \cos 7 \tau+c_{92} \sin 9 \tau+d_{92} \cos 9 \tau
\end{aligned}
$$

Note that in the expression for $X_{12}$, there is no $\cos \tau$ term to maintain consistency with the first approximation and its $\mathrm{O}(\varepsilon)$ refinement. Equations (27), (29) and (33) are then substituted into (26) and harmonic balance is then carried out, leading to a set of linear equations for $a_{12}, a_{32}, b_{32}, a_{52}, b_{52}, a_{72}$, $b_{72}, a_{92}, b_{92}, c_{12}, d_{12}, c_{32}, d_{32}, c_{52}, d_{52}, c_{72}, d_{72}, c_{92}, d_{92}$, and $\omega_{2}$. In doing so, it will be seen that $a_{12}, a_{32}, b_{32}$, $c_{12}, d_{12}, c_{32}, d_{32}$, and $\omega_{2}$ will all be non-zero, so that there will now be corrections to the frequency together with first and third harmonics. The following equations are then obtained for the coefficients:

$$
\begin{gathered}
\left(H_{11}-\omega_{0}^{2}\right) a_{12}+H_{12} c_{12}-\omega_{0} G_{12} d_{12}-\left(G_{12} d_{10}+2 \omega_{0} a_{10}\right) \omega_{2}+3 \mu S_{11}=0 \\
\omega_{0} G_{11} a_{12}+\omega_{0} G_{12} c_{12}+H_{12} d_{12}+\left(G_{12} c_{10}+G_{11} a_{10}\right) \omega_{2}+3 \mu C_{11}=0 \\
H_{21} a_{12}+\left(H_{22}-\omega_{0}^{2}\right) c_{12}-\omega_{0} G_{22} d_{12}-\left(2 \omega_{0} c_{10}+G_{22} d_{10}\right) \omega_{2}=0 \\
\omega_{0} G_{21} a_{12}+\omega_{0} G_{22} c_{12}+\left(H_{22}-\omega_{0}^{2}\right) d_{12}-\left(2 \omega_{0} d_{10}-G_{22} c_{10}-G_{21} a_{10}\right) \omega_{2}=0
\end{gathered}
$$

$3 \omega_{0} G_{11} a_{32}+\left(H_{11}-9 \omega_{0}^{2}\right) b_{32}+3 \omega_{0} G_{12} c_{32}+H_{12} d_{32}$ $=-3 \mu C_{31}+18 \omega_{2} \omega_{0} b_{30}-3 G_{11} \omega_{2} a_{30}-3 G_{12} \omega_{2} c_{30}$

$H_{21} a_{32}-3 \omega_{0} G_{21} b_{32}+\left(H_{22}-9 \omega_{0}^{2}\right) c_{32}-3 \omega_{0} G_{22} d_{32}$

$$
=18 \omega_{2} \omega_{0} c_{30}+3 b_{30} G_{21} \omega_{2}+3 G_{22} \omega_{2} d_{30}
$$

$3 \omega_{0} G_{21} a_{32}+H_{21} b_{32}+3 \omega_{0} G_{22} c_{32}+\left(H_{22}-9 \omega_{0}^{2}\right) d_{32}$

$=18 \omega_{2} \omega_{0} d_{30}-3 a_{30} G_{21} \omega_{2}-3 G_{22} \omega_{2} c_{30}$

$$
\begin{gathered}
\left(H_{11}-25 \omega_{0}^{2}\right) a_{52}-5 \omega_{0} G_{11} b_{52}+H_{12} c_{52}-5 \omega_{0} G_{12} d_{52}=-3 \mu S_{51} \\
5 \omega_{0} G_{11} a_{52}+\left(H_{11}-25 \omega_{0}^{2}\right) b_{52}+5 \omega_{0} G_{12} c_{52}+H_{12} d_{52}=-3 \mu C_{51} \\
H_{21} a_{52}-5 \omega_{0} G_{21} b_{52}+\left(H_{22}-25 \omega_{0}^{2}\right) c_{52}-5 \omega_{0} G_{22} d_{52}=0 \\
5 \omega_{0} G_{21} a_{52}+H_{21} b_{52}+5 \omega_{0} G_{22} c_{52}+\left(H_{22}-25 \omega_{0}^{2}\right) d_{52}=0
\end{gathered}
$$




$$
\begin{gathered}
\left(H_{11}-49 \omega_{0}^{2}\right) a_{72}-7 \omega_{0} G_{11} b_{72}+H_{12} c_{72}-7 \omega_{0} G_{12} d_{72}=-3 \mu S_{71} \\
7 \omega_{0} G_{11} a_{72}+\left(H_{11}-49 \omega_{0}^{2}\right) b_{72}+7 \omega_{0} G_{12} c_{72}+H_{12} d_{72}=-3 \mu C_{71} \\
H_{21} a_{72}-7 \omega_{0} G_{21} b_{72}+\left(H_{22}-49 \omega_{0}^{2}\right) c_{72}-7 \omega_{0} G_{22} d_{72}=0 \\
7 \omega_{0} G_{21} a_{72}+H_{21} b_{72}+7 \omega_{0} G_{22} c_{72}+\left(H_{22}-49 \omega_{0}^{2}\right) d_{72}=0 \\
\\
\left(H_{11}-81 \omega_{0}^{2}\right) a_{92}-9 \omega_{0} G_{11} b_{92}+H_{12} c_{92}-9 \omega_{0} G_{12} d_{92}=-3 \mu S_{91} \\
9 \omega_{0} G_{11} a_{92}+\left(H_{11}-81 \omega_{0}^{2}\right) b_{92}+9 \omega_{0} G_{12} c_{92}+H_{12} d_{92}=-3 \mu C_{91} \\
H_{21} a_{92}-9 \omega_{0} G_{21} b_{92}+\left(H_{22}-81 \omega_{0}^{2}\right) c_{92}-9 \omega_{0} G_{22} d_{92}=0 \\
9 \omega_{0} G_{21} a_{92}+H_{21} b_{92}+9 \omega_{0} G_{22} c_{92}+\left(H_{22}-81 \omega_{0}^{2}\right) d_{92}=0
\end{gathered}
$$

where $C_{11}, S_{11}, C_{31}, S_{31}, C_{51}, S_{51}, C_{71}, S_{71}, C_{91}, S_{91}$ are given in Appendix $\mathrm{C}$ and will be known in terms of $a_{10}, a_{30}, b_{30}, c_{10}, d_{10}, c_{30}, d_{30}, a_{11}, a_{31}, b_{31}, a_{51}, b_{51}, a_{71}, b_{71}, a_{91}, b_{91}, c_{11}, d_{11}, c_{31}, d_{31}, c_{51}, d_{51}, c_{71}, d_{71}$, $c_{91}, d_{91}$.

The refined approximation is then obtained from $X_{1}=X_{10}+X_{11}+X_{12}, X_{2}=X_{20}+X_{21}+X_{22}$ (noting that $\varepsilon$ is now set to 1 as discussed previously).

\section{Stability Analysis}

The stability of the limit cycles obtained by the method in Section 3 are now determined by Floquet analysis. The implementation adopted is that used in [23], which in turn was an extension of an approach developed in [34] for the analysis of Chua's circuit, a first order three degree-of-freedom system. It is now assumed that $\mathbf{X}$ represents a limit cycle oscillation of (2) and $\Delta \mathbf{X}$ a small perturbation of the limit cycle. Then to first order, $\Delta \mathbf{X}$, satisfies the equation:

$$
\Delta \ddot{\mathbf{X}}+\mathbf{G} \Delta \dot{\mathbf{X}}+\mathbf{H} \Delta \mathbf{X}+3 \mu\left(\begin{array}{l}
1 \\
0
\end{array}\right) X_{1}^{2} \Delta \mathbf{X}=\mathbf{0}
$$

where $\mathbf{G}$ and $\mathbf{H}$ are the $2 \times 2$ damping and stiffness matrices whose elements are $G_{\mathrm{ij}}$ and $H_{\mathrm{ij}}$ (where i,j $=1,2$ ) respectively.

A solution for $\Delta \mathbf{X}$ is now sought by writing:

$$
\Delta \mathbf{X}=\sum_{l=1}^{4} H_{l} \mathbf{v}_{l}(t) e^{\lambda_{l} t}
$$

where $H_{l}$ are scalar constants whose values would be dependent on initial conditions, $\boldsymbol{v}_{1}$ are twodimensional vector functions of period $2 \pi / \omega$ and the $\lambda_{1}$ are complex numbers which will determine the stability of the limit cycle. The $\lambda_{1}$ 's may then be related to the Floquet multipliers $\mu_{1}$ by the Equation:

$$
\mu_{l}=\exp \left(\frac{2 \pi \lambda_{l}}{\omega}\right)
$$

For a periodic solution one value of $\mu_{1}$ will always be unity, and therefore the nature of the remaining three $\mu_{1}$ values will determine the stability of the limit cycle and will also give an indication of the type of bifurcation occurring if the magnitude of one of the three $\mu_{1}$ 's exceeds unity. Full details of the stability analysis process may be found in [23]. 


\section{Limit Cycle Analysis}

In this section, examples of limit cycle predictions are given and comparisons with time domain results are made. In the time domain approach, Equations (2) are solved numerically using a variable step Runge-Kutta method. All the examples are based on an aeroelastic analysis of an all-moving control surface with a nonlinearity in the torsional degree-of-freedom of the root support. Supersonic airflow was considered, and the aerodynamic loadings were modelled using third order piston theory aerodynamics [35]. Because of the simple form of the aerodynamics arising from the use of piston theory, the resulting aeroelastic equations take the form of Equations (2) with the matrices $\mathbf{G}$ and $\mathbf{H}$ being asymmetric and combining aerodynamic and linear structural damping and stiffness terms. Displacements of the control surface are expressed in terms of its modes of vibration in the absence of the nonlinearity so that $\mathbf{X}$ is a vector of generalised displacements.

In this example, $\mathbf{G}$ and $\mathbf{H}$ are given by:

$$
\begin{gathered}
\mathbf{G}=\left[\begin{array}{cc}
0.01915544+2.320517 \mathrm{C} & 0.001287775 \\
0.0001988401 & 0.01741002
\end{array}\right] \\
\mathbf{H}=\left[\begin{array}{ccc}
0.258710+0.128916 K & 1.389352 \\
0.214524 & 1.152059+0.0236412 K
\end{array}\right]+V\left[\begin{array}{cc}
-0.01634809 & 0.0007149875 \\
-0.03607494 & 0.004045464
\end{array}\right]
\end{gathered}
$$

The nonlinearity parameter $\mu$ will be re-written as:

$$
\mu=0.1289176 \delta K
$$

where $C$ and $K$ correspond to linear damping and stiffness terms and $V$ is a speed parameter. The numerical values were obtained for a control surface of cropped delta planform, with the natural modes, frequencies and corresponding generalised masses and stiffnesses being obtained from a finite element model composed of shell elements with a scalar spring element providing bending stiffness in the root support. For the piston theory aerodynamics, sea level international standard atmosphere conditions were assumed.

$\mu$ will be taken as positive throughout (signifying a hardening nonlinearity) and characterises a deviation from the linear torsional stiffness $K$.

An indication of some of the bifurcational behaviour of the system that may typically be expected is shown in Fig. 1 where amplitudes $X_{1 \max }$ of limit cycle response in terms of the variable $X_{1}$ as $V$ is increased from 0 to 9.0 are presented for $K=1.0$ and $\delta=2.5$ for values of $C$ of $0.05,0.35$ and 0.5 . Results have been obtained by both the second refined approximation and time domain analysis supported by a linear stability analysis for the equilibrium point $X_{1}=X_{2}=0$. In each case, the pattern of behaviour is similar. For low values of $V$, the only attractor is the stable equilibrium at $X_{1}=X_{2}=0$, and at a certain value of $V$, dependent on $C$, a limit cycle pair appear. In each case, the upper branch shown in Fig. 1 corresponds to a stable limit cycle and the lower branch to an unstable limit cycle. The stability is shown by the Floquet multipliers obtained, and examples of these are shown in Table 1. As $V$ increases further, the unstable limit cycle disappears in a subcritical Hopf bifurcation, and so the equilibrium point $X_{1}=X_{2}=0$ becomes unstable while the amplitude of the stable limit cycle continues to grow. It may also be seen that increasing $C$ delays appearance of the limit cycles and that the amplitude of the stable limit cycle increases with $C$. The subcritical Hopf bifurcation occurs earlier as $C$ is initially increased from 0.05 , but beyond a certain value of $C$, the $V$ for which this occurs begins to increase. Fig. 1 gives an indication that good agreement can be obtained between time domain predictions and the method presented in this paper. Apart from presenting Floquet multipliers, Table 1 
also shows predictions of the limit cycle fundamental frequency obtained from both the second refined approximation and the time domain analysis, which show very good agreement.

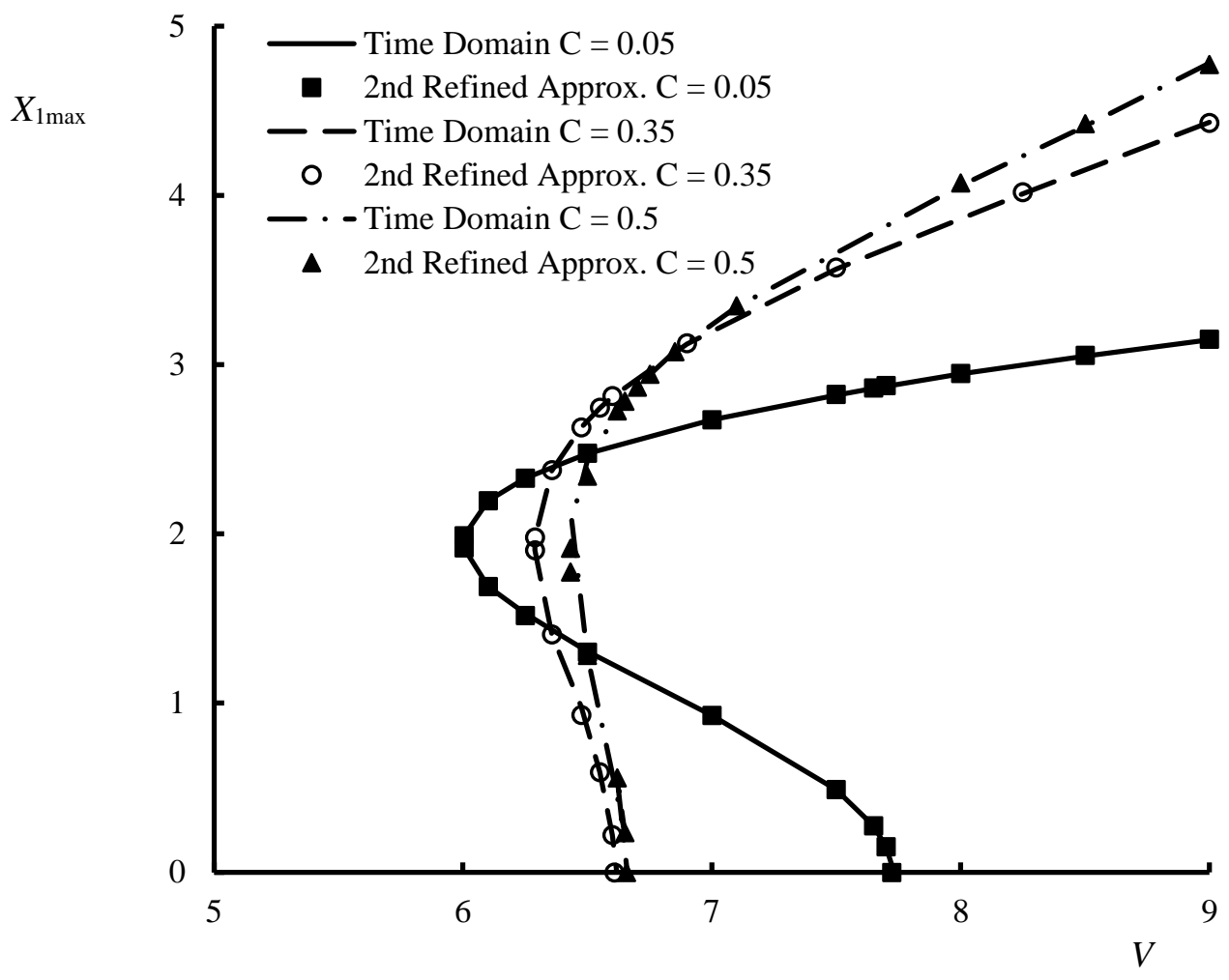

Fig. 1 Bifurcational Behaviour of Nonlinear System $-K=1.0$ and $\delta=2.5$

Table 1 Floquet Multipliers and Frequencies for Limit Cycles $-K=\mathbf{1 . 0}$ and $\delta=\mathbf{2 . 5}$

\begin{tabular}{|c|c|c|c|c|c|}
\hline$C$ & $V$ & Floquet Multipliers & Stability & $\begin{array}{c}\text { Frequency }-2^{\text {nd }} \\
\text { Refined } \\
\text { Approximation }\end{array}$ & $\begin{array}{l}\text { Frequency - } \\
\text { Time Domain }\end{array}$ \\
\hline \multirow{5}{*}{0.05} & 6.5 & $\begin{array}{lcc}0.9176 & -0.4497 \pm 0.5074 \mathrm{i} \\
2.5701 & 0.8357 & 0.1882\end{array}$ & $\begin{array}{l}\text { Stable } \\
\text { Unstable }\end{array}$ & $\begin{array}{l}1.1105 \\
1.0585\end{array}$ & $\begin{array}{l}1.1106 \\
1.0584\end{array}$ \\
\hline & 7.0 & $\begin{array}{rr}0.9194 & -0.6472 \pm 0.2095 \mathrm{i} \\
1.3522 & 0.4161 \pm 0.3510 \mathrm{i}\end{array}$ & $\begin{array}{c}\text { Stable } \\
\text { Unstable }\end{array}$ & $\begin{array}{l}1.1218 \\
1.0483\end{array}$ & $\begin{array}{l}1.1218 \\
1.0483\end{array}$ \\
\hline & 7.5 & $\begin{array}{lll}0.9207 & -0.7973 & -0.5833 \\
1.0253 & -0.2963 \pm 0.5480 \mathrm{i}\end{array}$ & $\begin{array}{l}\text { Stable } \\
\text { Unstable }\end{array}$ & $\begin{array}{l}1.1304 \\
1.0407\end{array}$ & $\begin{array}{l}1.1304 \\
1.0407\end{array}$ \\
\hline & 8.0 & $0.9218-0.6667 \pm 0.1502 \mathrm{i}$ & Stable & 1.1378 & 1.1378 \\
\hline & 9.0 & $0.9240-0.5375 \pm 0.4257 \mathrm{i}$ & Stable & 1.1501 & 1.1500 \\
\hline \multirow{5}{*}{0.35} & 6.475 & $\begin{array}{ll}0.9400 & -0.0786 \pm 0.0430 \mathrm{i} \\
1.0291 & -0.0231 \pm 0.0800 \mathrm{i}\end{array}$ & $\begin{array}{l}\text { Stable } \\
\text { Unstable }\end{array}$ & $\begin{array}{l}1.0915 \\
1.0788\end{array}$ & $\begin{array}{l}1.0915 \\
1.0788\end{array}$ \\
\hline & 6.55 & $\begin{array}{ll}0.9378 & -0.0899 \pm 0.0033 \mathrm{i} \\
1.0106 & -0.0836 \pm 0.0064 \mathrm{i}\end{array}$ & $\begin{array}{c}\text { Stable } \\
\text { Unstable }\end{array}$ & $\begin{array}{l}1.0928 \\
1.0778\end{array}$ & $\begin{array}{l}1.0928 \\
1.0778\end{array}$ \\
\hline & 7.5 & $\begin{array}{lll}0.9378 & 0.1656 & 0.0510 \\
\end{array}$ & Stable & 1.1023 & 1.1022 \\
\hline & 8.25 & 0.5092 & Stable & 1.1069 & 1.1066 \\
\hline & 9.0 & 0.7040 & Stable & 1.1105 & 1.1097 \\
\hline
\end{tabular}


In the following study, the effectiveness of the approximation method of Sections 2 and 3 will now be investigated. Interest will focus on the behaviour of the stable limit cycles indicated in Fig. 1 as $C, K$ and $V$ are varied, and as the nonlinear effect becomes more significant. Limit cycle predictions of Section 2 based on first and third harmonics will be referred to as 'first approximation' and those of Section 3, where higher harmonic effects are now included will be referred to as 'first refined approximation' and 'second refined approximation'. In the following, results will first be presented in terms of $X_{1}$ against $\dot{X_{1}}$.

As a first example, limit cycle predictions are shown in Figs. 2 to 5 for the case $K=1.0, V=9.0, \delta=$ 2.5 for four values of damping parameter $C$ and compare time domain predictions with the first and refined approximations. For $C=0.0$, as shown in Fig. 2, the first approximation gives good agreement with the time domain result. For $C=0.05$, as shown in Fig. 3, agreement between first approximation and time domain predictions can be seen to be beginning to deteriorate but the first refined approximation gives an improvement on the first approximation. For the higher values of $C$ considered in Figs. 4 and 5, it may be seen that the second refined approximation gives improvements on both the first approximation and its first refinement. Floquet analysis indicated that the limit cycles are stable for the values of $C$ considered and this was confirmed by the time domain analysis. A similar set of predictions are shown in Figs. 6 to 9 for the for the case $K=4.0, V=10.0, \delta=2.5$ for four values of damping parameter $C$. For $C=0.0$, as shown in Fig. 6, the first approximation gives good agreement with time domain results. For $C=0.15$, as shown in Fig. 7, agreement between first approximation and time domain predictions can be seen to be beginning to deteriorate but the first refined approximation gives an improvement on the first approximation. For the higher values of $C$ considered in Figs. 8 and 9 , it may be seen that the second refined approximation gives improvements on both the first approximation and its first refinement.

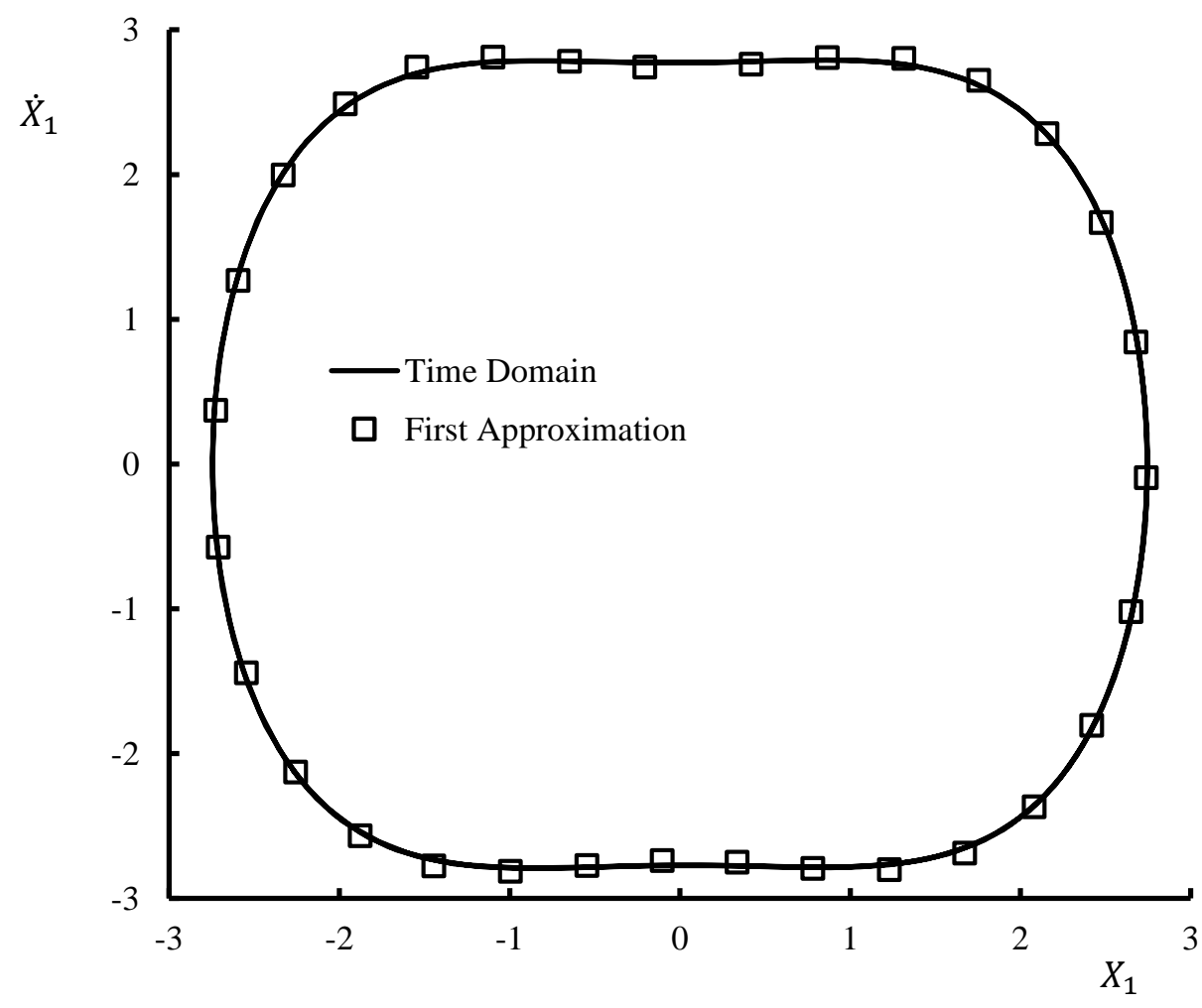

Fig. 2 Limit Cycle Predictions. $K=1.0, V=9.0, C=0.0, \delta=2.5$ 


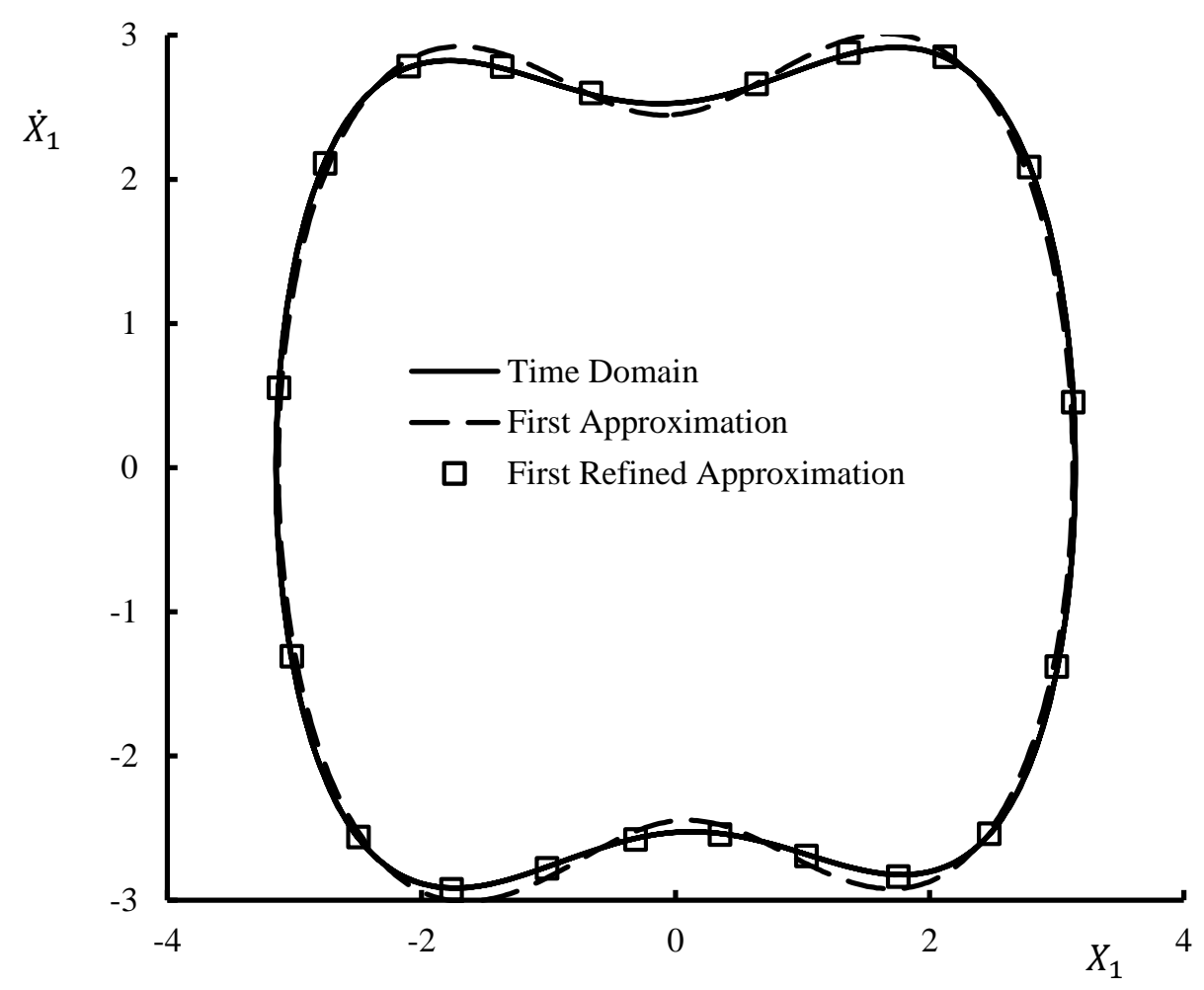

Fig. 3 Limit Cycle Predictions. $K=1.0, V=9.0, C=0.05, \delta=2.5$

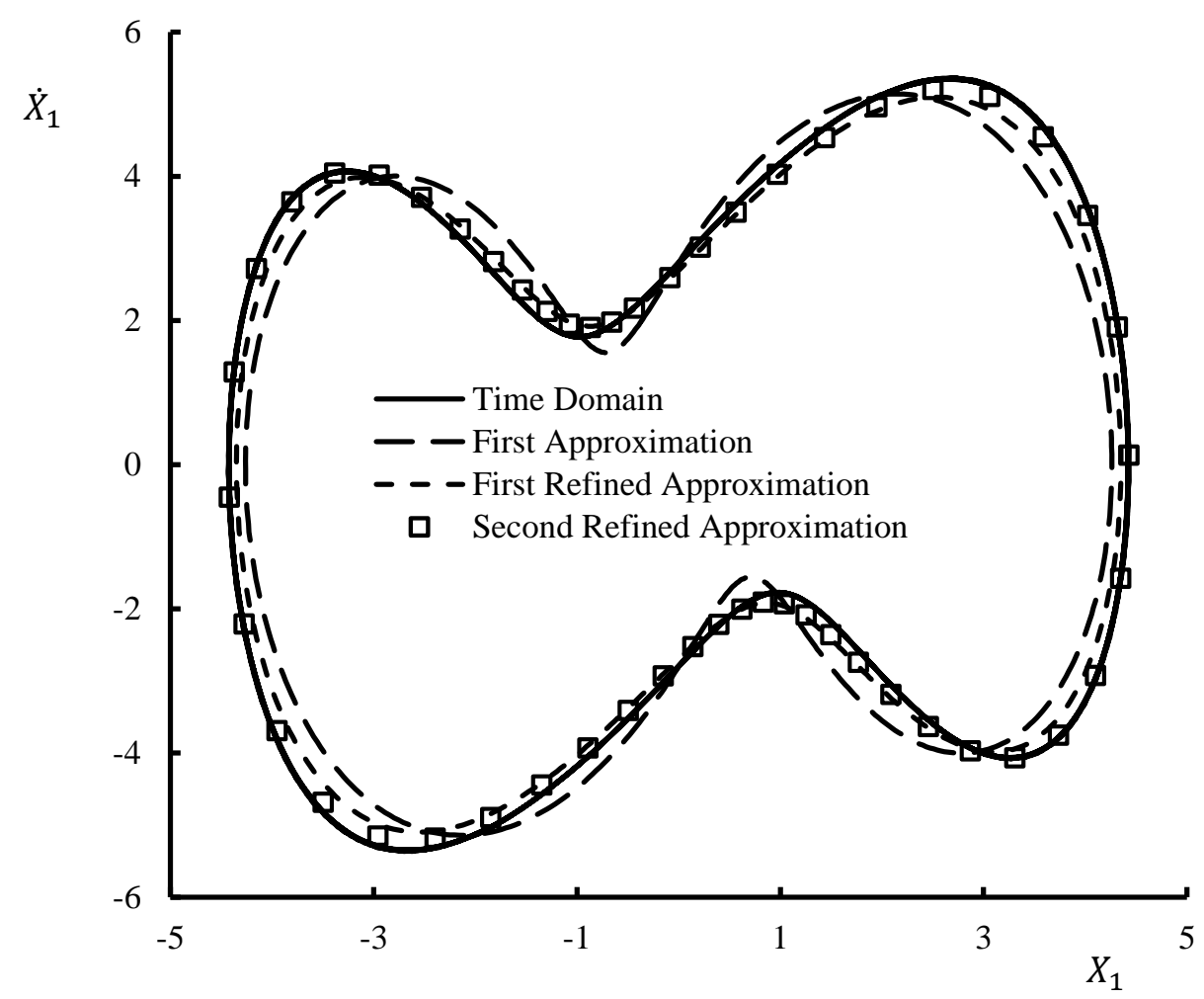

Fig. 4 Limit Cycle Predictions. $K=1.0, V=9.0, C=0.35, \delta=2.5$ 


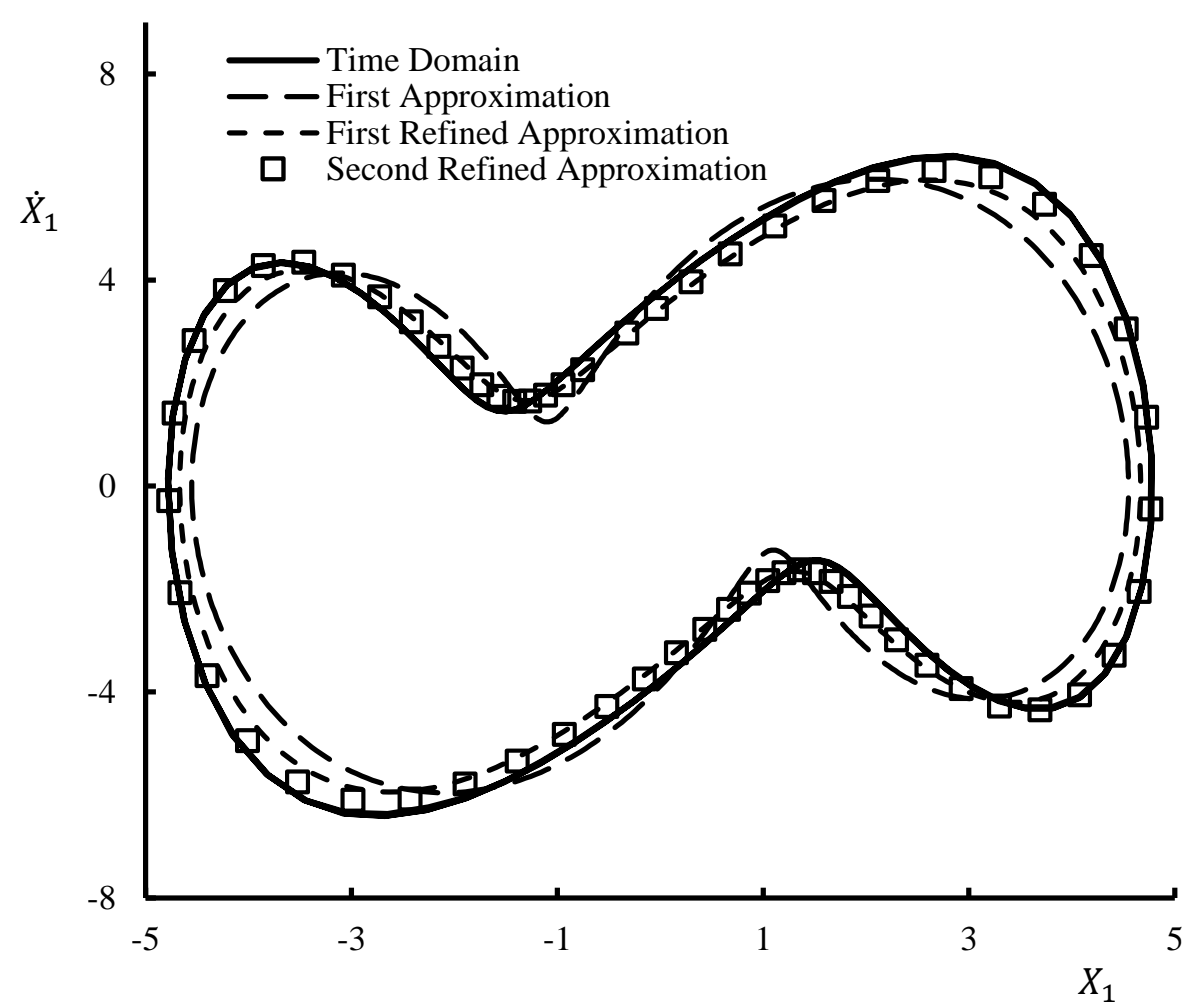

Fig. 5 Limit Cycle Predictions. $K=1.0, V=9.0, C=0.5, \delta=2.5$

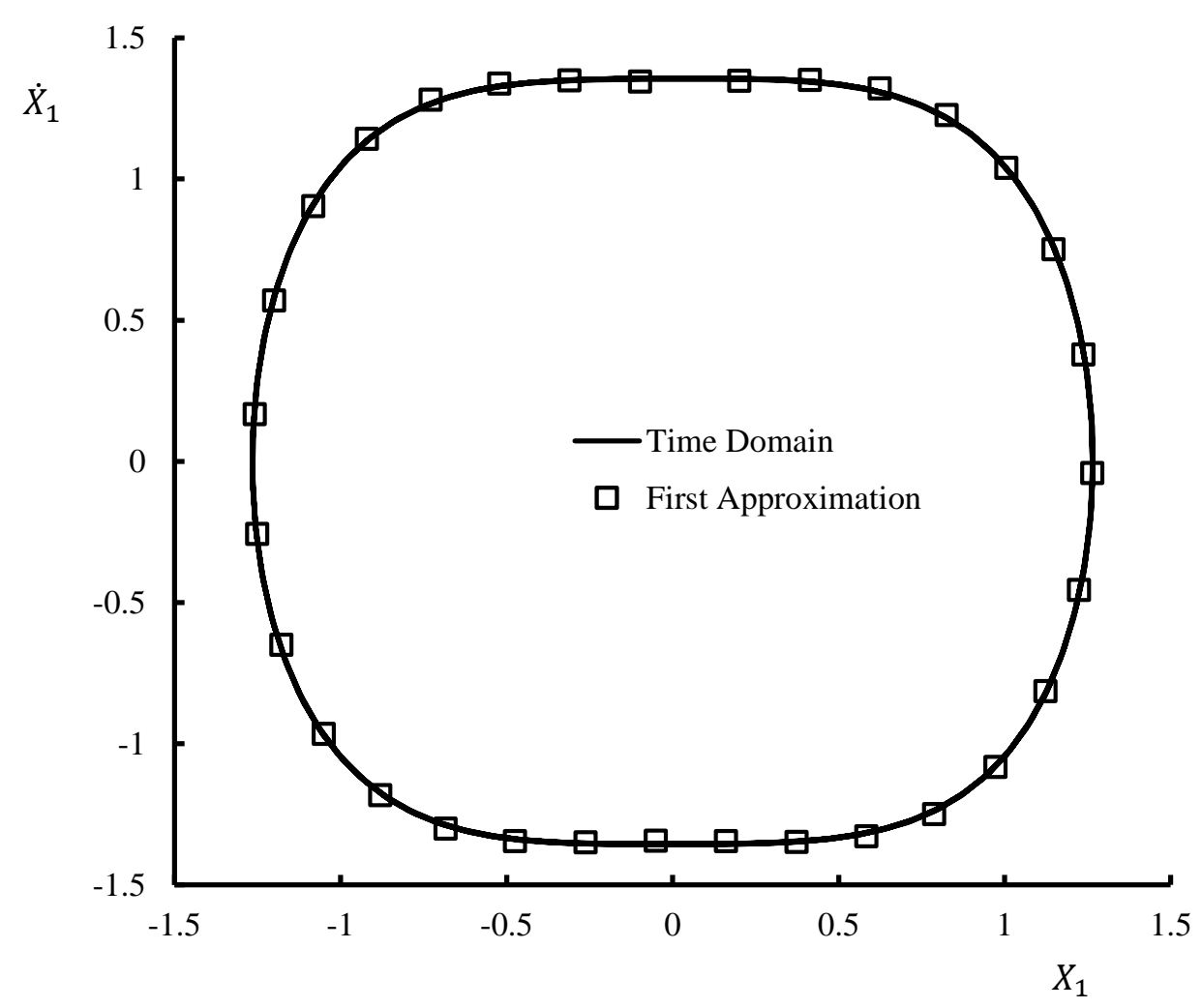

Fig. 6 Limit Cycle Predictions. $K=4.0, V=10.0, C=0.0, \delta=2.5$ 


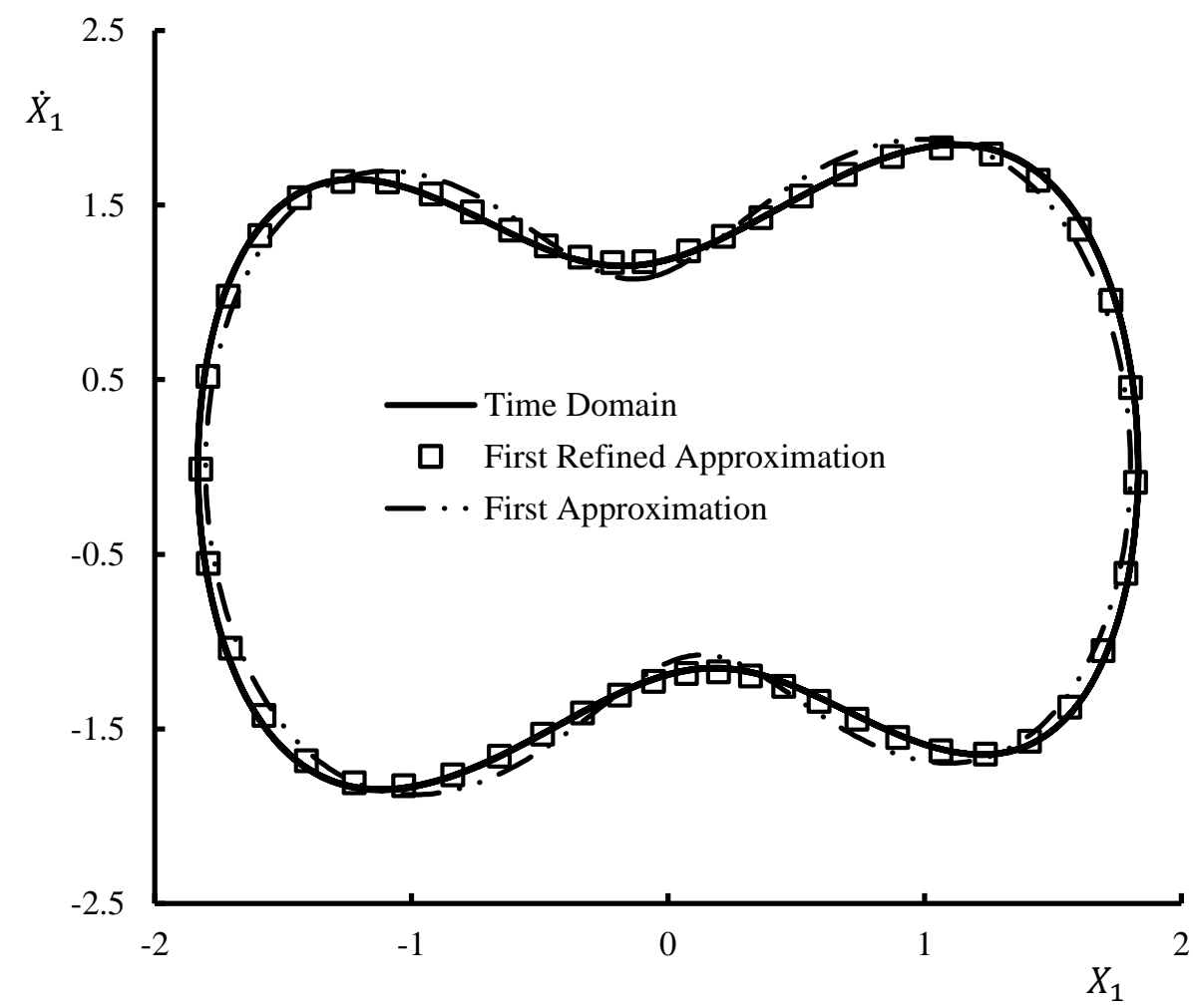

Fig. 7 Limit Cycle Predictions. $K=4.0, V=10.0, C=0.15, \delta=2.5$

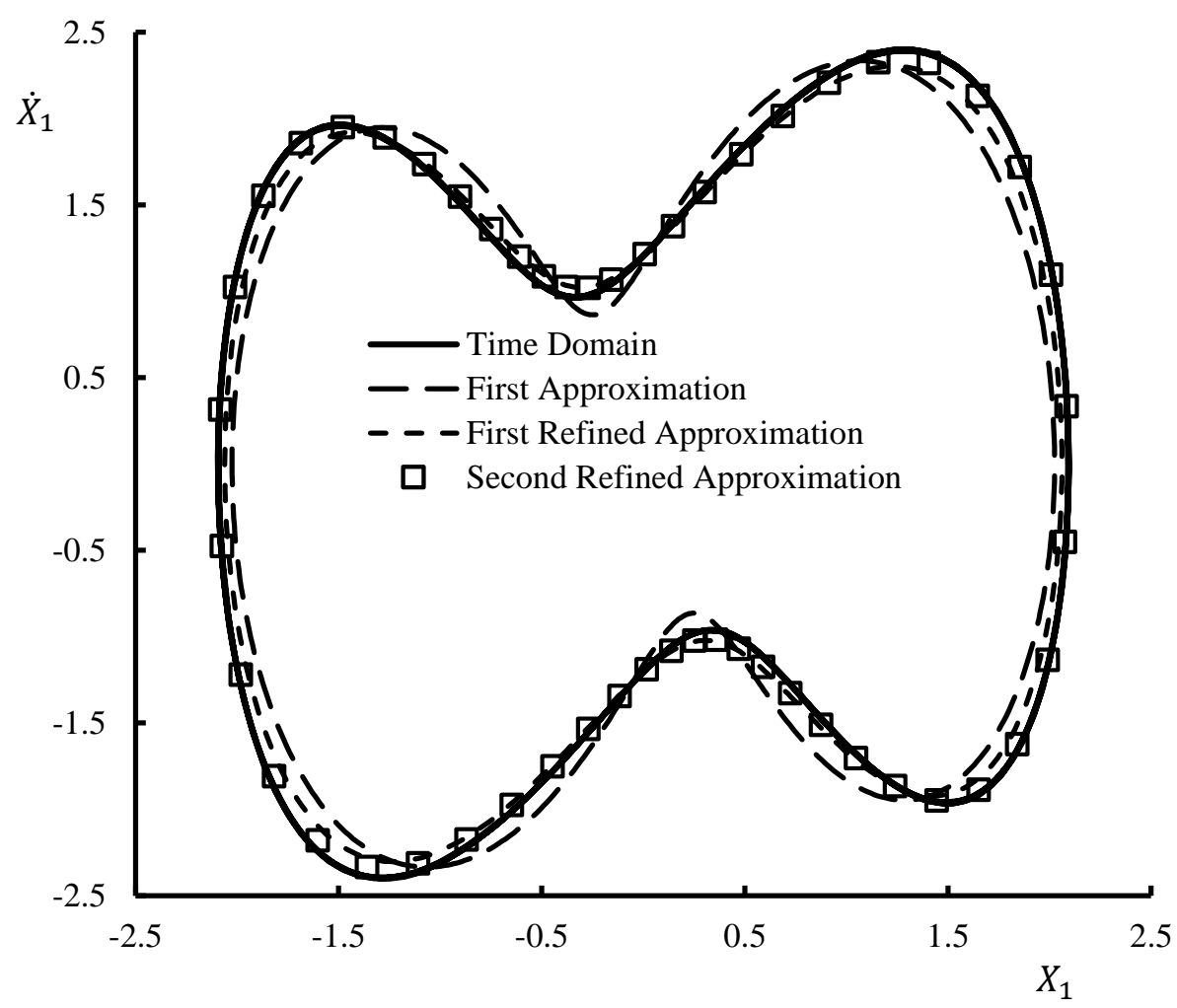

Fig. 8 Limit Cycle Predictions. $K=4.0, V=10.0, C=0.25, \delta=2.5$ 


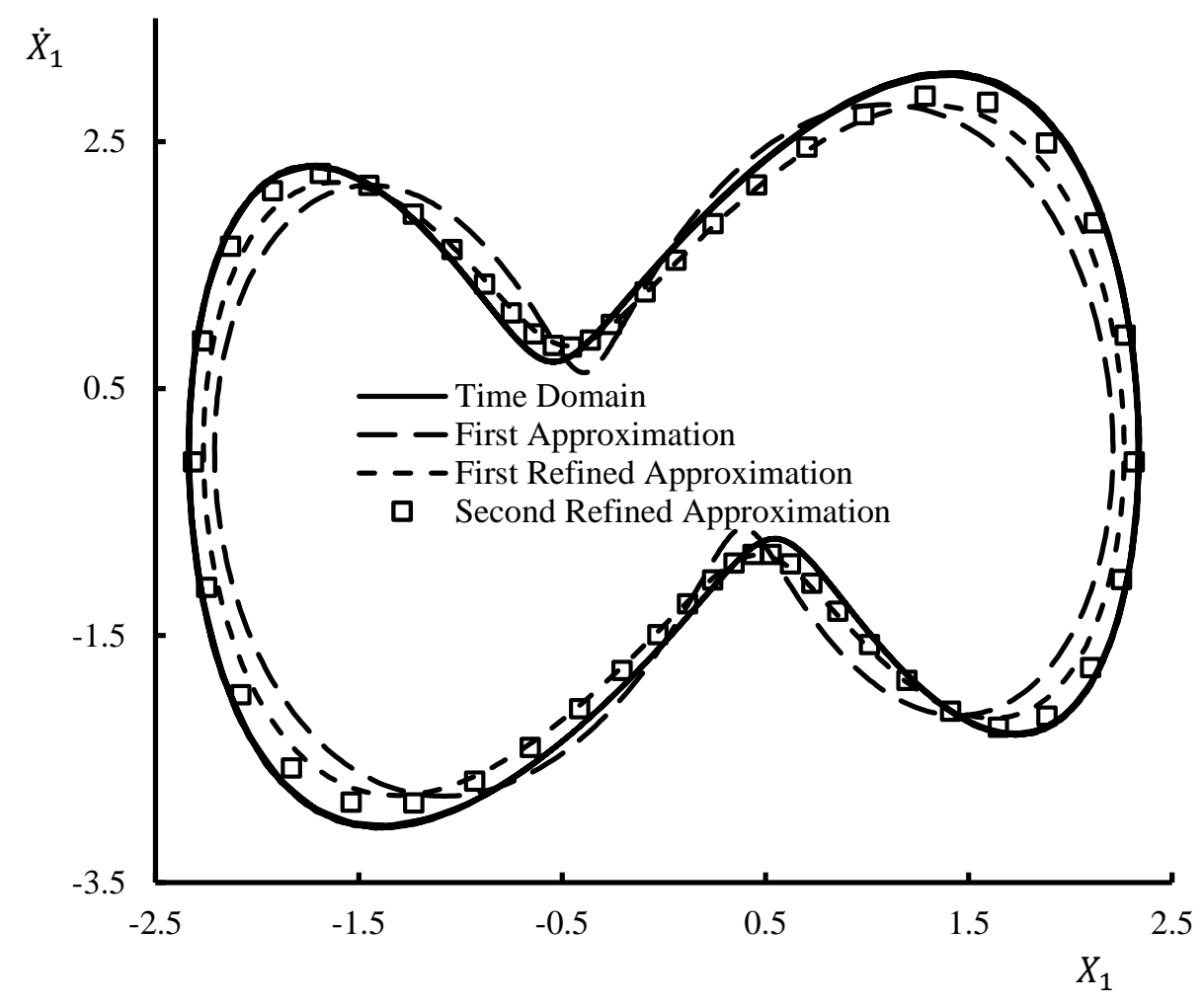

Fig. 9 Limit Cycle Predictions. $K=4.0, V=10.0, C=0.35, \delta=2.5$

As another way of comparing the predictions, amplitudes of the harmonic components of the motion were obtained in the time domain by using a Discrete Fourier Transform, ensuring that the sample time history used is taken over complete cycles. The Discrete Fourier Transform (DFT) and refined approximation predictions of the amplitudes of the harmonics are compared in Figs. 10 to 15 for the cases $K=1.0, V=9.0, \delta=2.5$ and $K=4.0, V=10.0, \delta=2.5$. The plots compare amplitudes of the first, third and fifth harmonics for time domain and the three approximations. It should be noted that for the first and third harmonics, the first and first refined approximations are the same, whereas for the fifth harmonic predictions, it should be recalled that the first approximation only includes first and third harmonics. Figs. 10 and 13 show very good agreement between the DFT and the two approximations for the first harmonic, although for the first approximation, agreement is marginally better. Figures 11, 12, 14 and 15 show how for the third and fifth harmonics, the refined approximations give increasing improvements. The effect of these refinements is the overall improvements demonstrated in Figs. 2 to 9. 


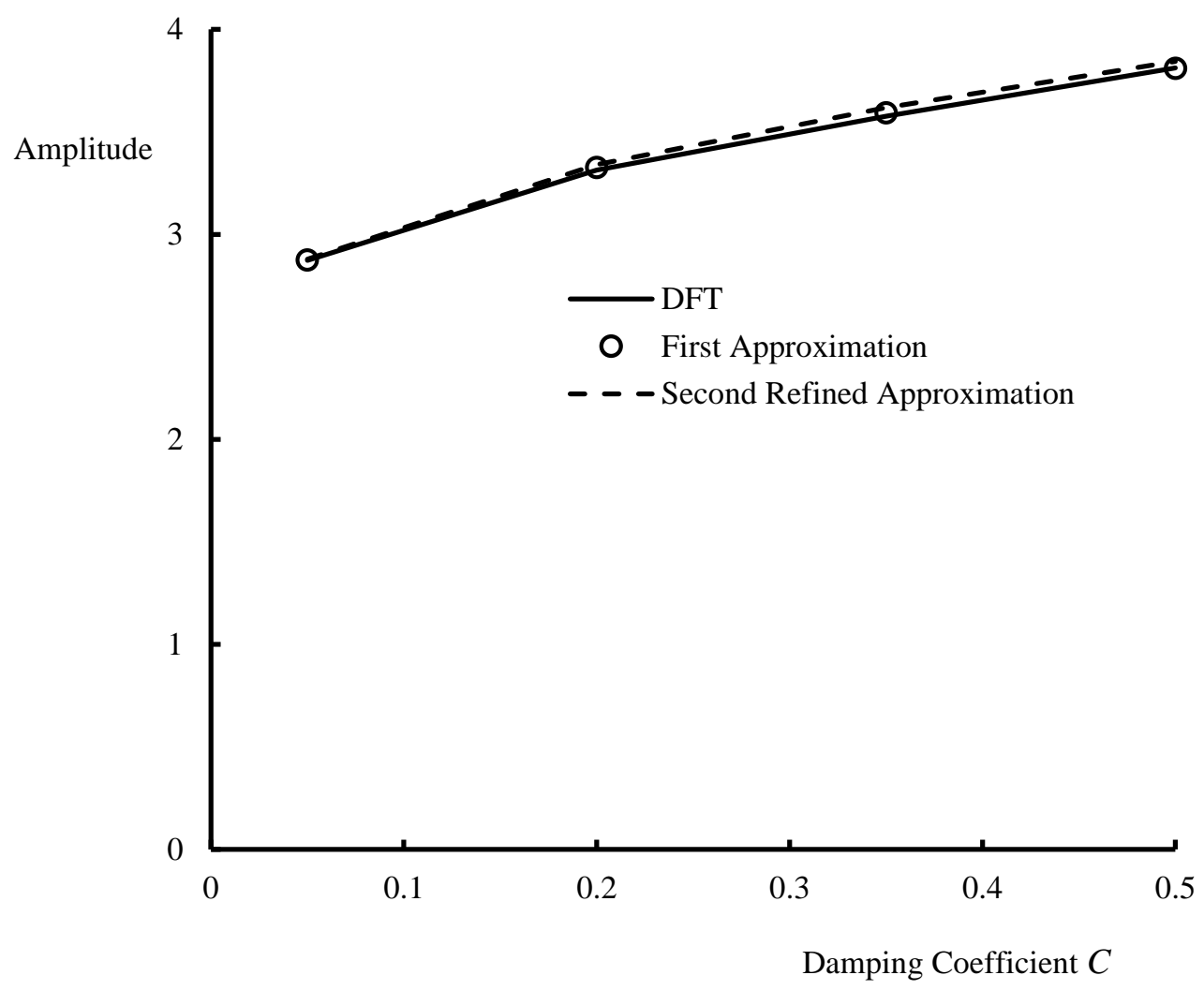

Fig. 10 Predictions of Amplitude of First Harmonic for $X_{1}, K=1.0, V=9.0, \delta=2.5$

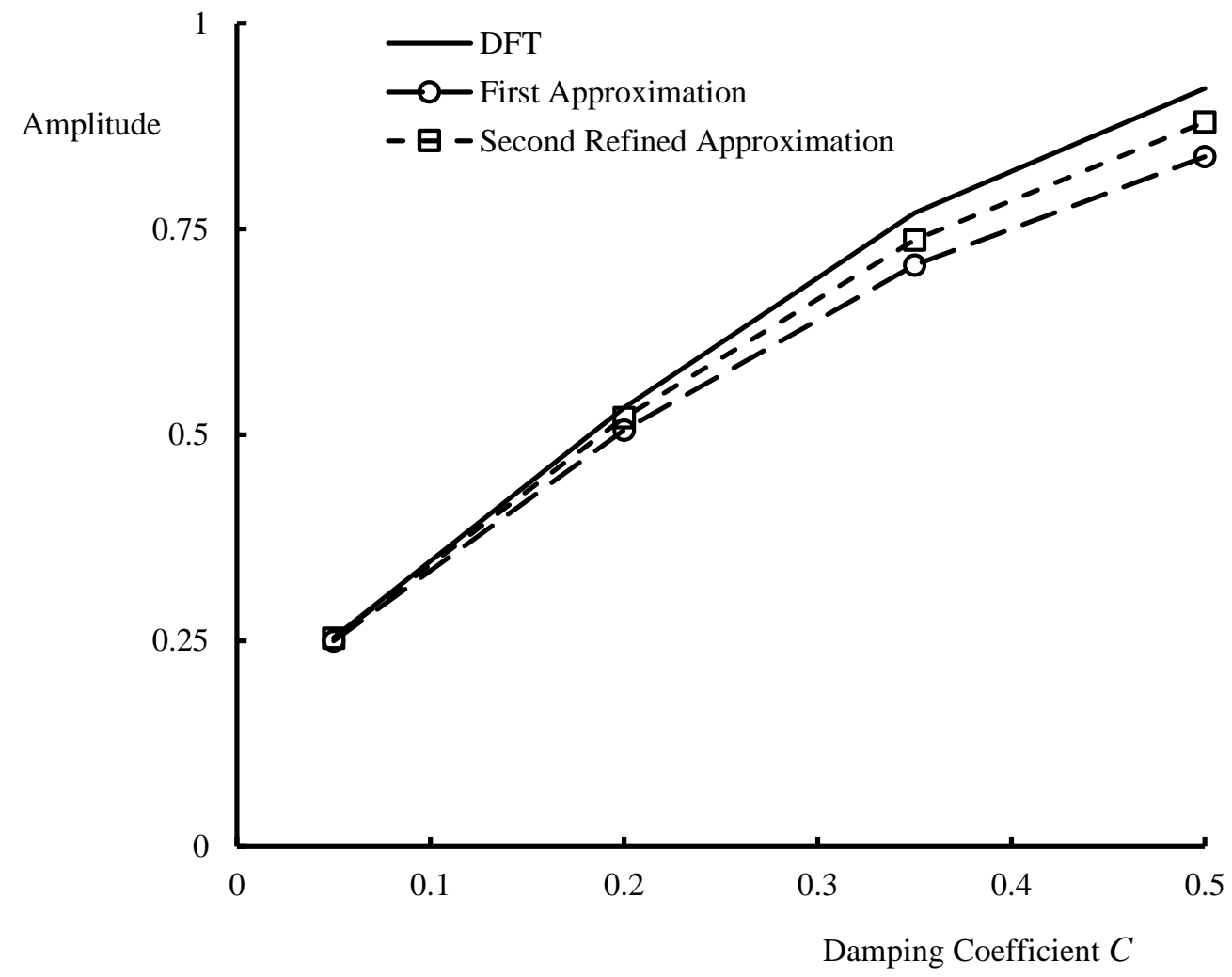

Fig. 11 Predictions of Amplitude of Third Harmonic for $X_{1}, K=1.0, V=9.0, \delta=2.5$ 


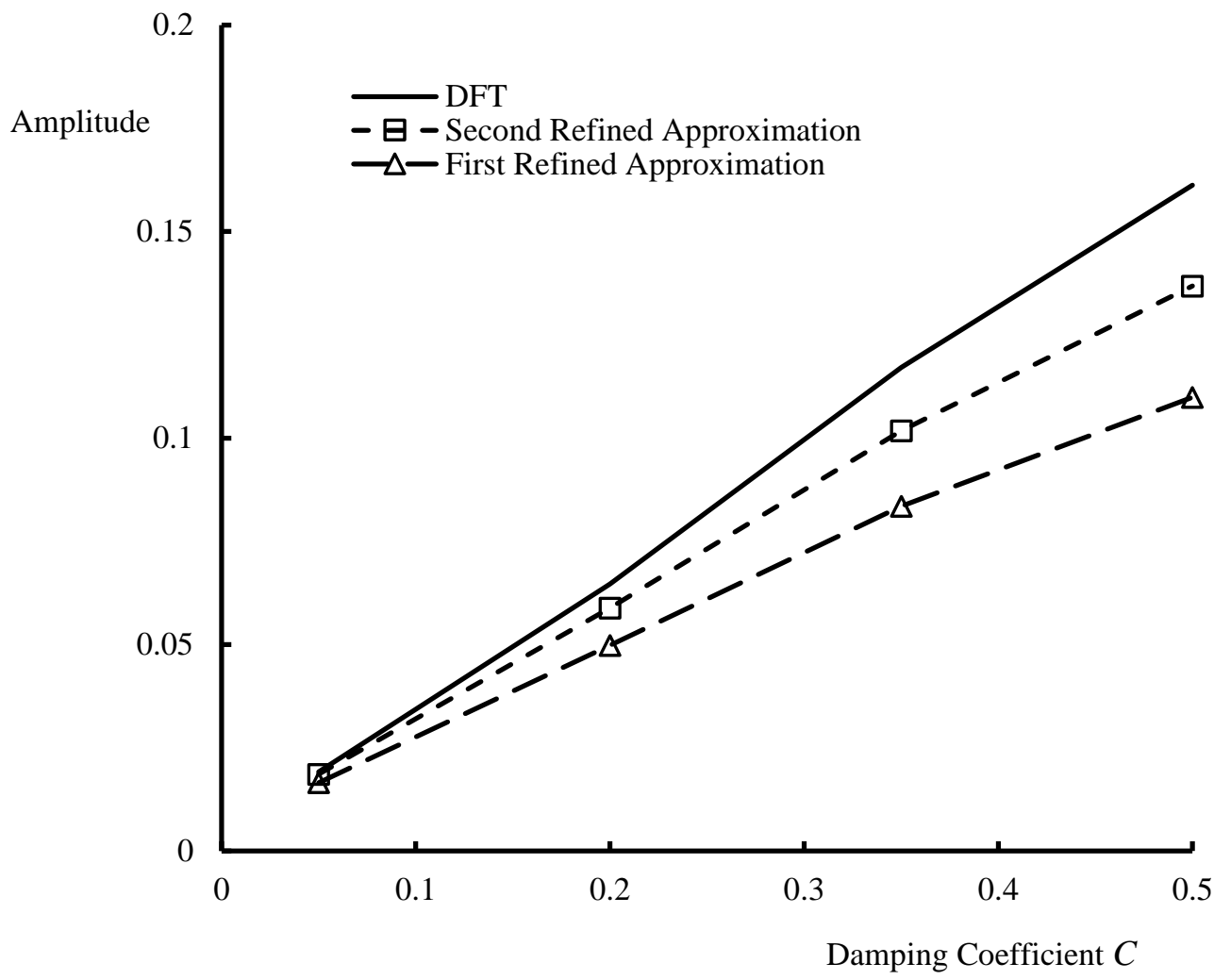

Fig. 12 Predictions of Amplitude of Fifth Harmonic for $X_{1}, K=1.0, V=9.0, \delta=2.5$

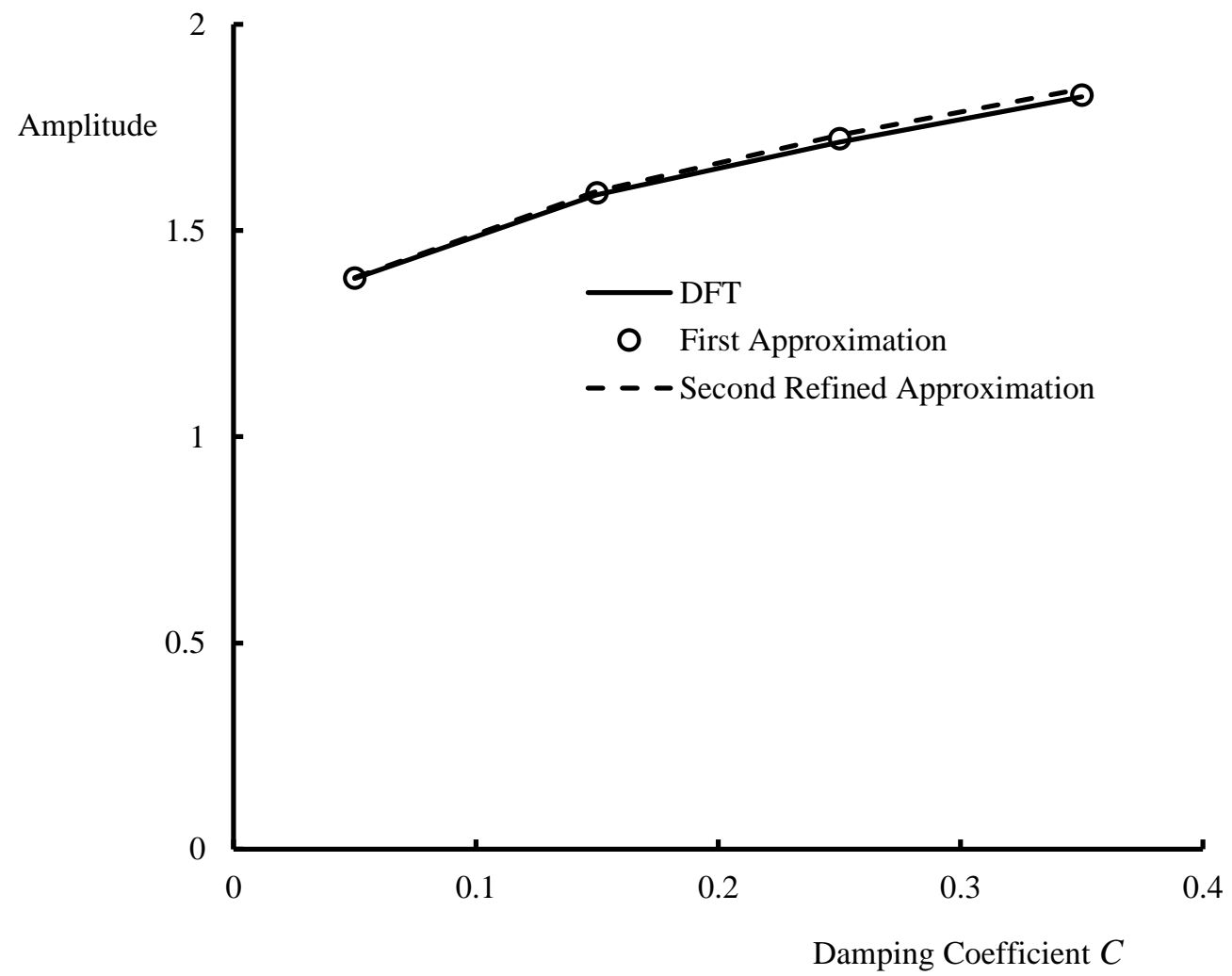

Fig. 13 Predictions of Amplitude of First Harmonic for $X_{1}, K=4.0, V=10.0, \delta=2.5$ 


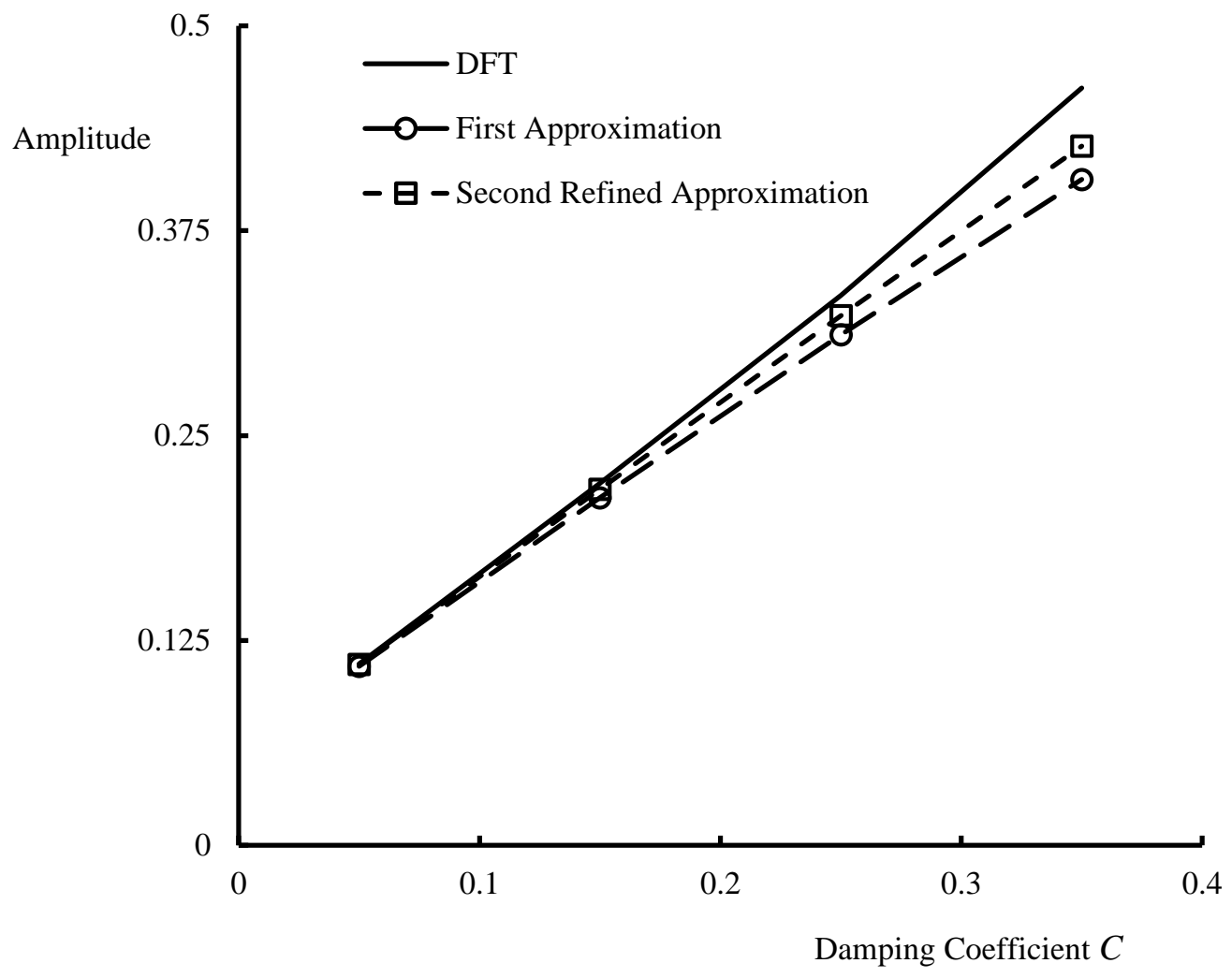

Fig. 14 Predictions of Amplitude of Third Harmonic for $X_{1}, K=4.0, V=10.0, \delta=2.5$

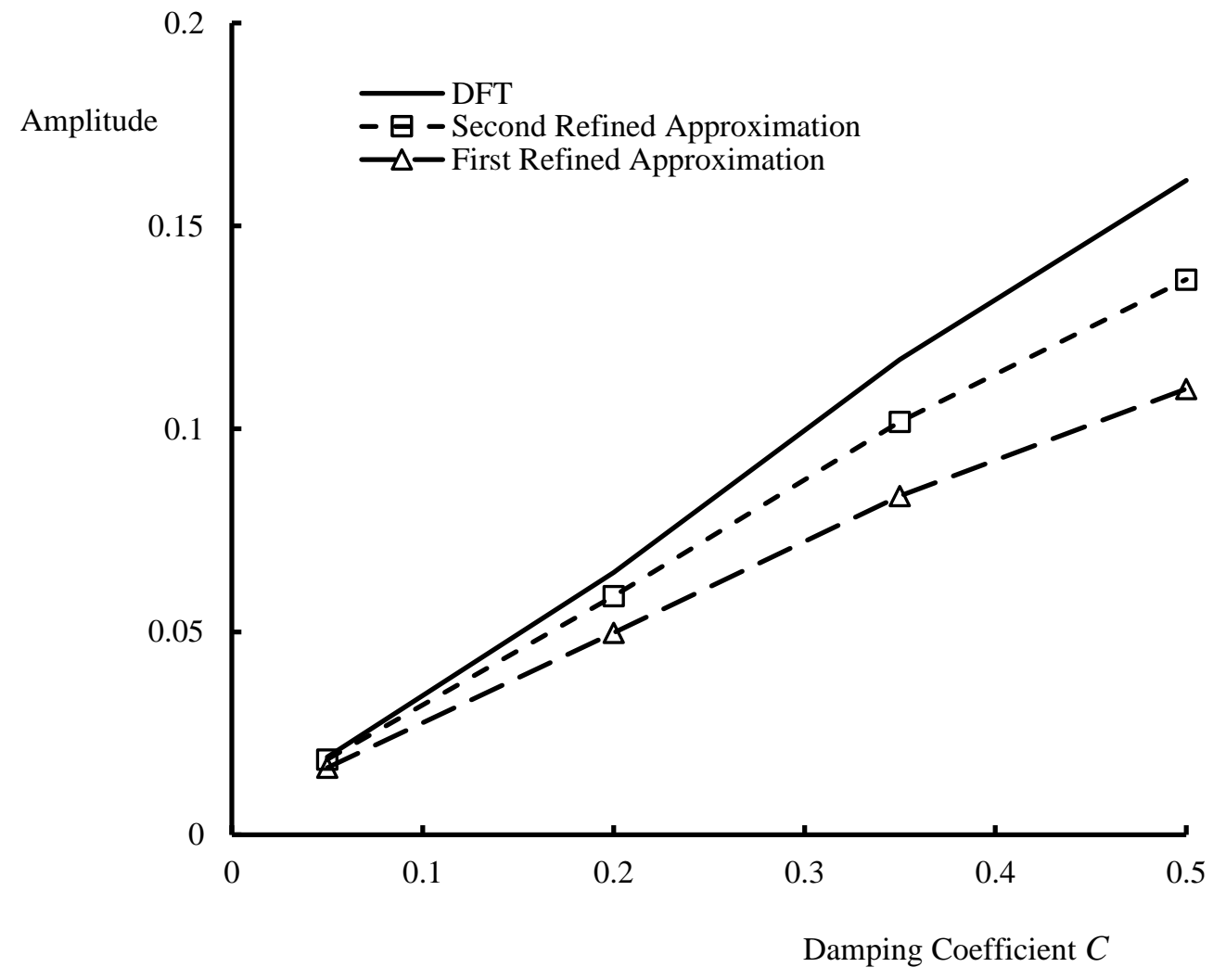

Fig. 15 Predictions of Amplitude of Fifth Harmonic for $X_{1}, K=4.0, V=10.0, \delta=2.5$ 
The results presented hitherto, have been in terms of $X_{1}$ against $\dot{X}_{1}$. Some results are now presented for $X_{2}$ against $\dot{X}_{2}$ in Figs. 16 to 18 . It was noted that for the range of parameters considered, the first harmonic dominated the motion, even in cases where higher harmonics were contributing significantly to $X_{1}$ and $\dot{X}_{1}$. An example of this is shown Fig. 16 for the case $K=4.0, V=10.0, C=0.25, \delta=2.5$ which compares $X_{2}$ and $\dot{X}_{2}$ as determined by time domain and the approximate method. As the first harmonic dominates the variation of $X_{2}$, further comparisons of predictions were made by comparing amplitudes of $X_{2}$. These are shown in Figs. 17 and 18 for cases $K=1.0, V=9.0, \delta=2.5$ and $K=4.0, V$ $=10.0, \delta=2.5$ respectively. Agreement between time domain predictions and the approximate methods is good. However, for higher displacements, the second refined approximation only gives slight improvement on the first approximation. It was also noted that the amplitudes of $X_{2}$ grows rapidly in comparison with the first harmonic component of $X_{1}$ as $C$ increases.

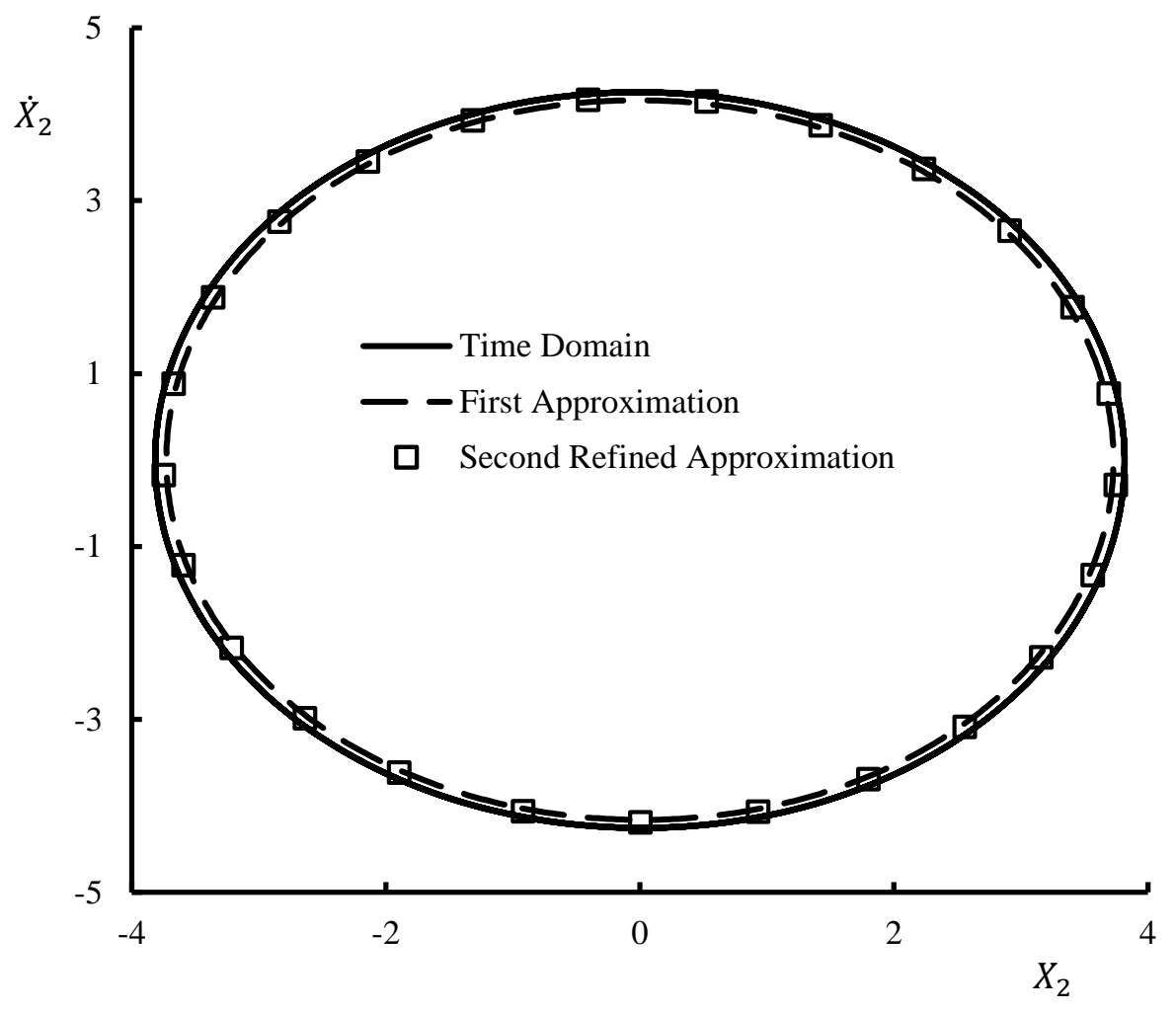

Fig. 16 Limit Cycle Prediction $-X_{2}$. and $\dot{X}_{2} . K=4.0, V=10.0, C=0.25, \delta=2.5$ 


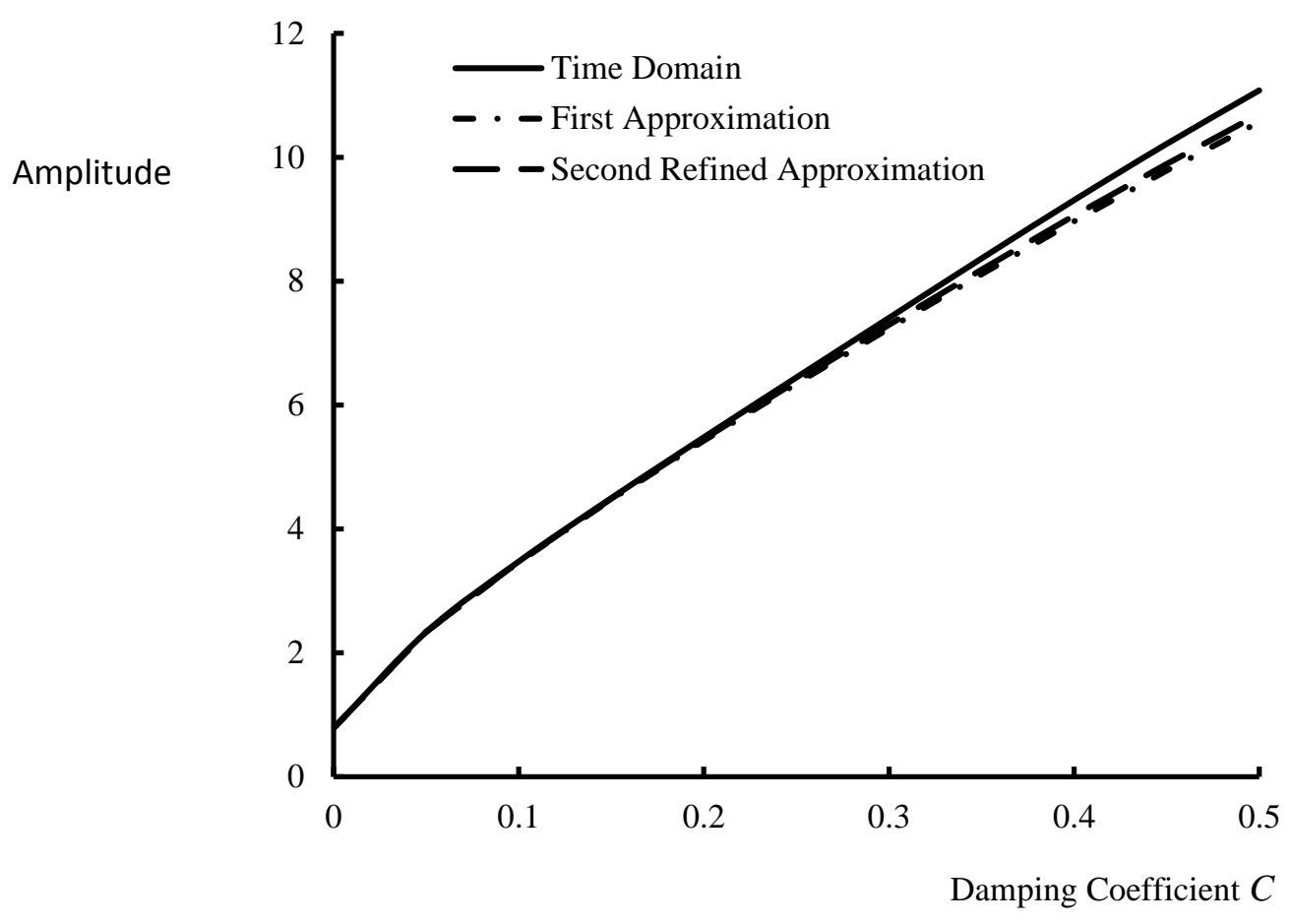

Fig. 17 Amplitude of $X_{2} \cdot K=1.0, V=9.0, \delta=2.5$

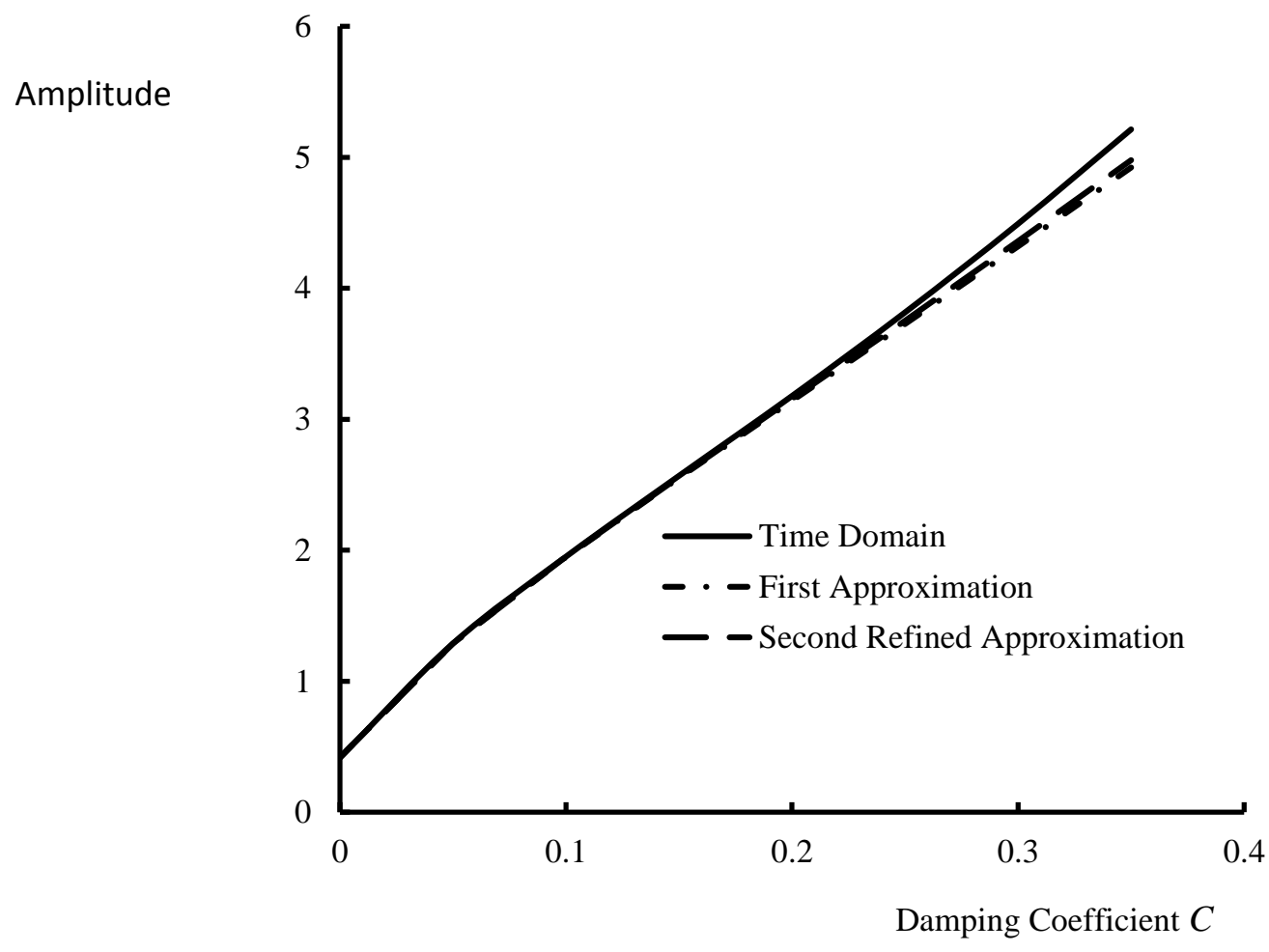

Fig. 18 Amplitude of $X_{2}^{-} K=4.0, V=10.0, \delta=2.5$ 
Investigations at higher values of $V$ were now carried out. One example of the results obtained is for the case $K=4, V=11.0, \delta=2.5$. Results for a $C$ value of 0.15 are presented in Fig. 19. As in previous examples, the refined approximation clearly leads to improved predictions for $X_{1}$. However, one of the Floquet multipliers for the limit cycle crosses the real axis and becomes greater than 1.0. This indicates that a fold (saddle-node), transcritical or pitchfork (symmetry-breaking) bifurcation of the limit cycle has occurred [36]. Time domain simulation confirmed that the limit cycle was unstable and further, there were now also two stable asymmetric limit cycles (involving even harmonics) showing that a symmetry-breaking bifurcation had occurred. These are shown in Fig. 20. The approximate methods would not be expected to predict these new limit cycles as they only assume odd harmonics make up the motion.

A study was now carried out to determine for a range of values of $C, K$ and $V$ where symmetry breaking occurred, Figs. 21 to 23 show comparisons of conditions for the occurrence of symmetry breaking bifurcations by both time domain analysis and the second refined approximation method. Agreement is good providing further verification of both the limit cycle prediction and Floquet multiplier calculation methods.

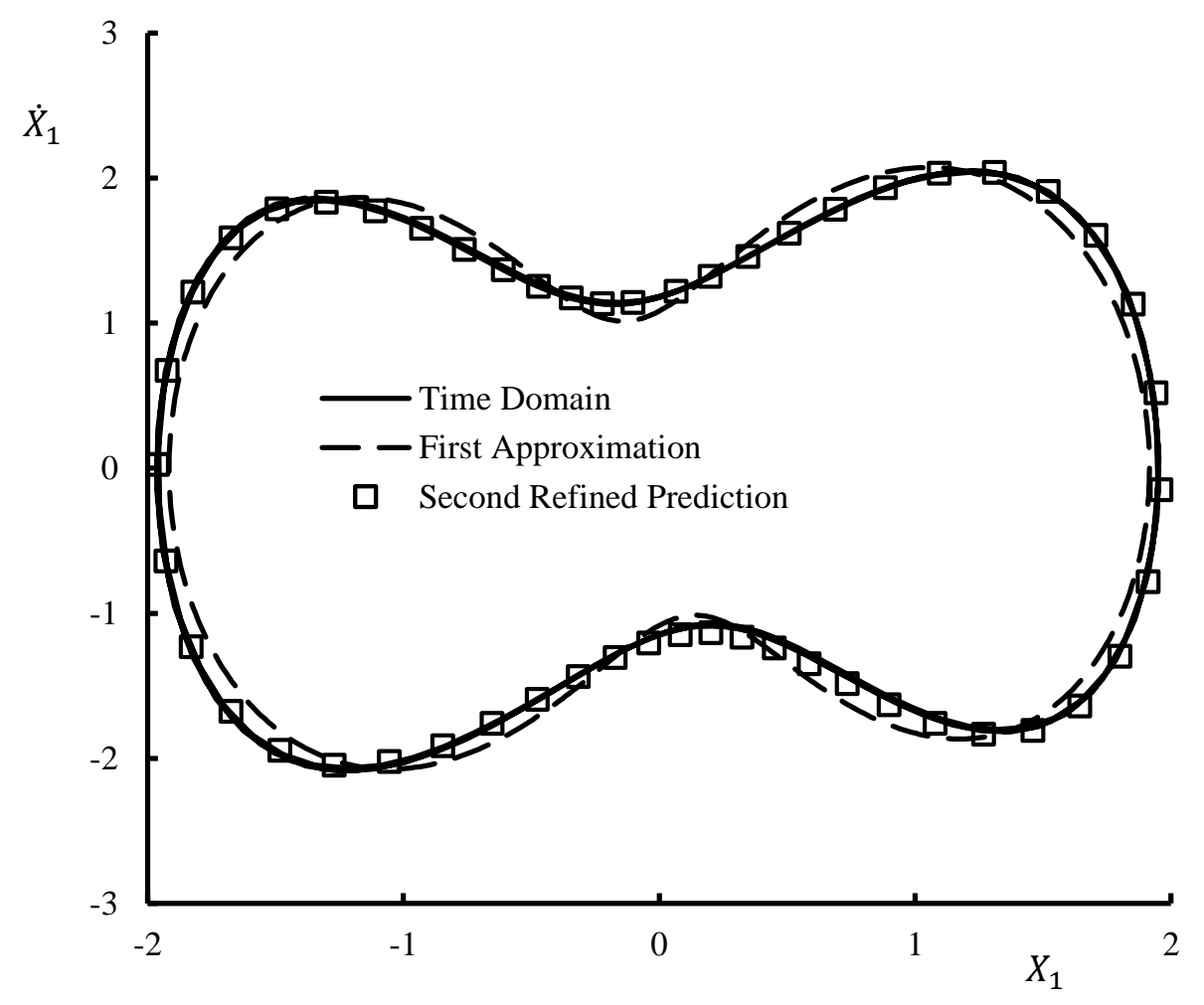

Fig. 19 Unstable Limit Cycle Prediction. $K=4.0, V=11.0, C=0.15, \delta=2.5$ 


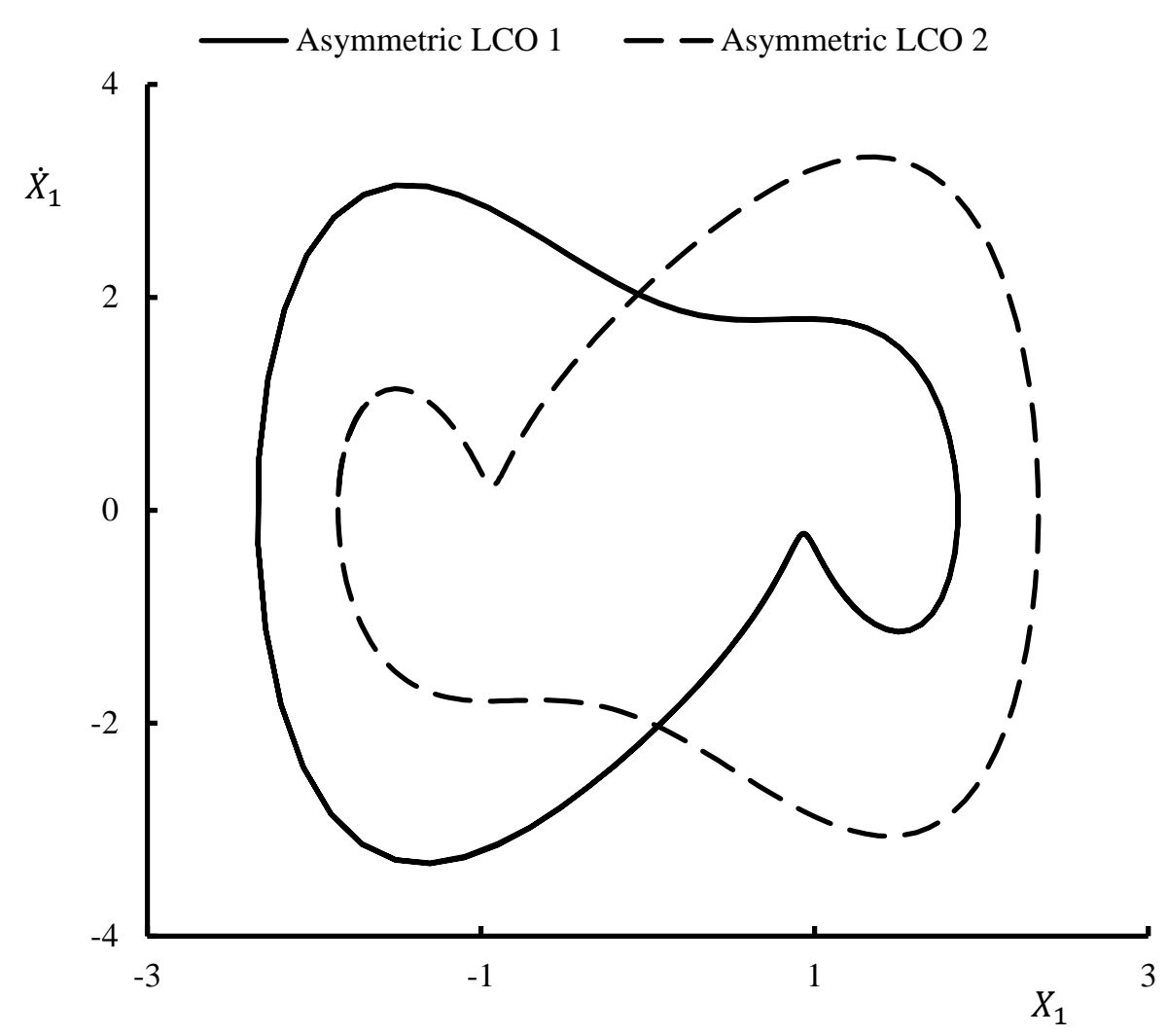

Fig. 20 Time Domain Predictions of Stable Asymmetric Limit Cycles. $K=4.0, V=11.0, C=$ $0.15, \delta=2.5$

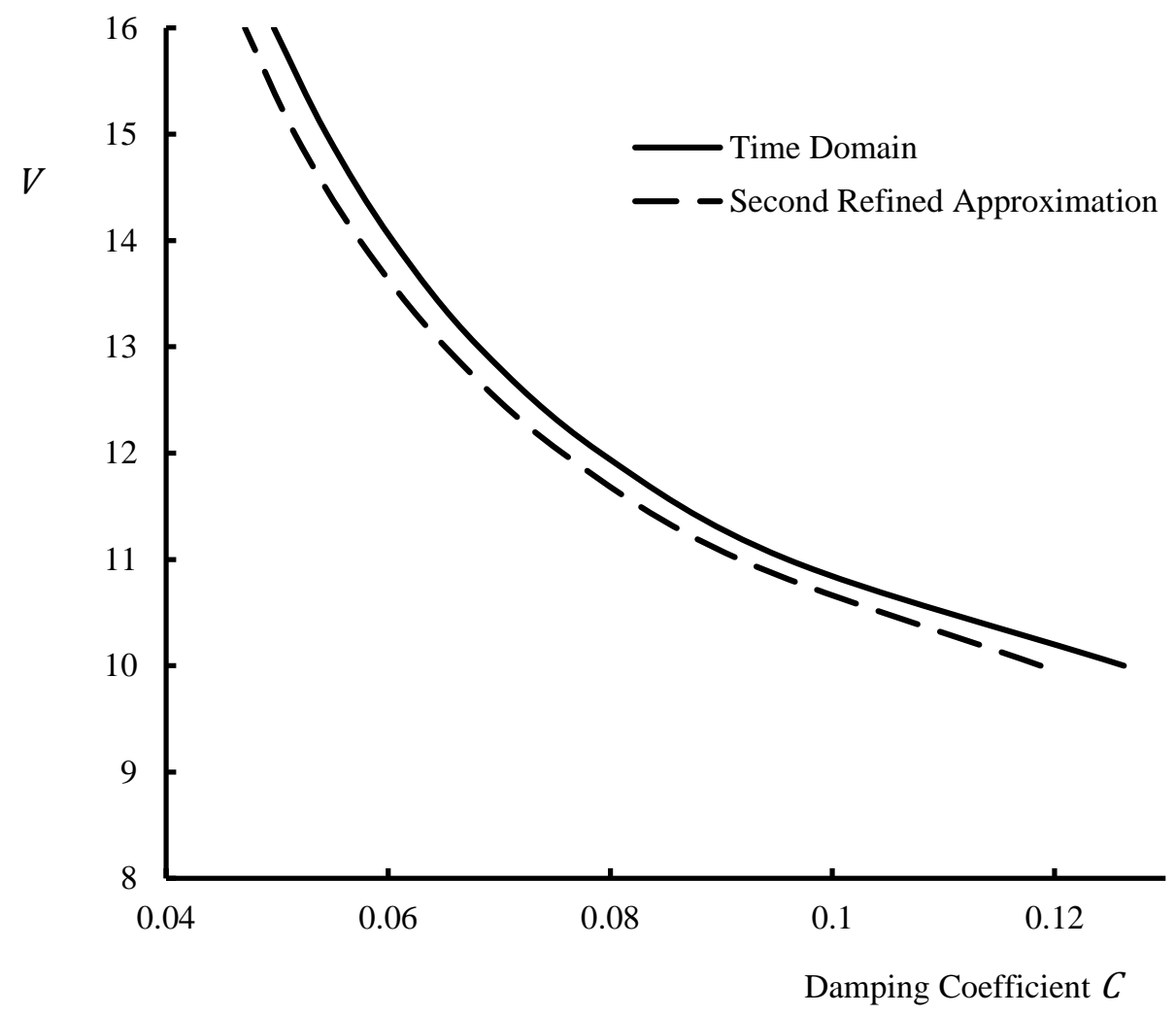

Fig. 21 Prediction of Onset of Limit Cycle Instability - $K=1, \delta=2.5$ 


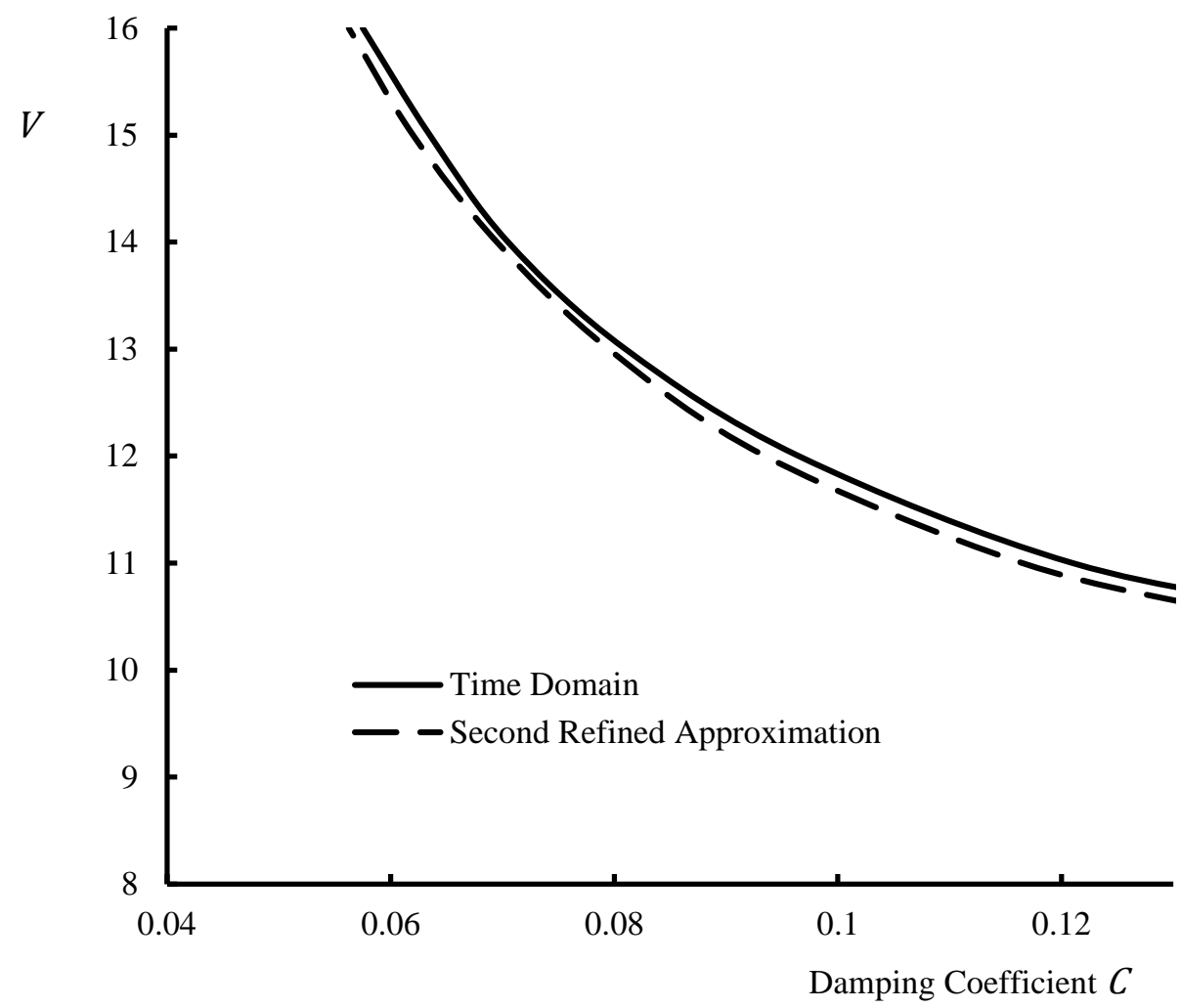

Fig. 22 Prediction of Onset of Limit Cycle Instability $-K=4, \delta=2.5$

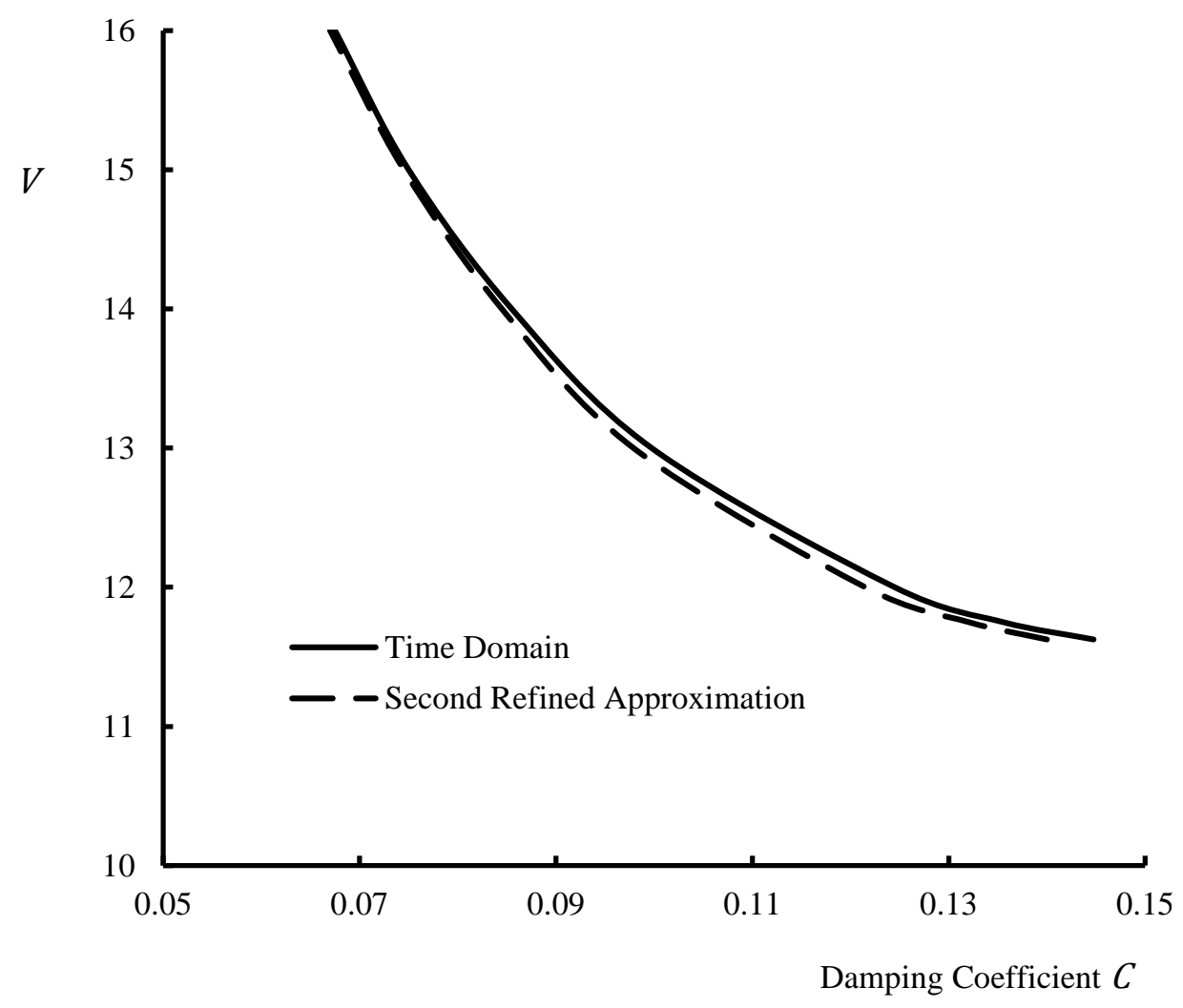

Fig. 23 Prediction of Onset of Limit Cycle Instability - $K=7, \delta=2.5$ 
A further bifurcation study was carried out for the case $K=4.0, C=0.1, \delta=2.5$ to illustrate more detail of the symmetry-breaking bifurcation that occurs, and this is shown in Fig. 24. As was done in Fig. 1, amplitudes $X_{1 \max }$ of limit cycle response in terms of the variable $X_{1}$ as $V$ is increased, in this case from 0 to 16.0 , are presented. Results have been obtained for symmetric limit cycles by both the second refined approximation and time domain analysis supported by a linear stability analysis for the equilibrium point $X_{1}=X_{2}=0$. Limit cycle predictions for asymmetric limit cycles are by time domain only. System behaviour as $V$ varies at first follows a similar pattern to that seen in Fig. 1.

Just before $V=12$, the symmetry breaking bifurcation occurs, as indicated in Fig. 24 by Point A. Beyond this point, along $\mathrm{AB}$, the symmetric limit cycle is unstable and two stable asymmetric limit cycles may be seen, and these are indicated by CD. However, when the bifurcation occurs, two unstable asymmetric limit cycles are produced, and these are indicated by AC. An example of two such asymmetric unstable limit cycles, determined in the time domain, are shown in Fig. 25.

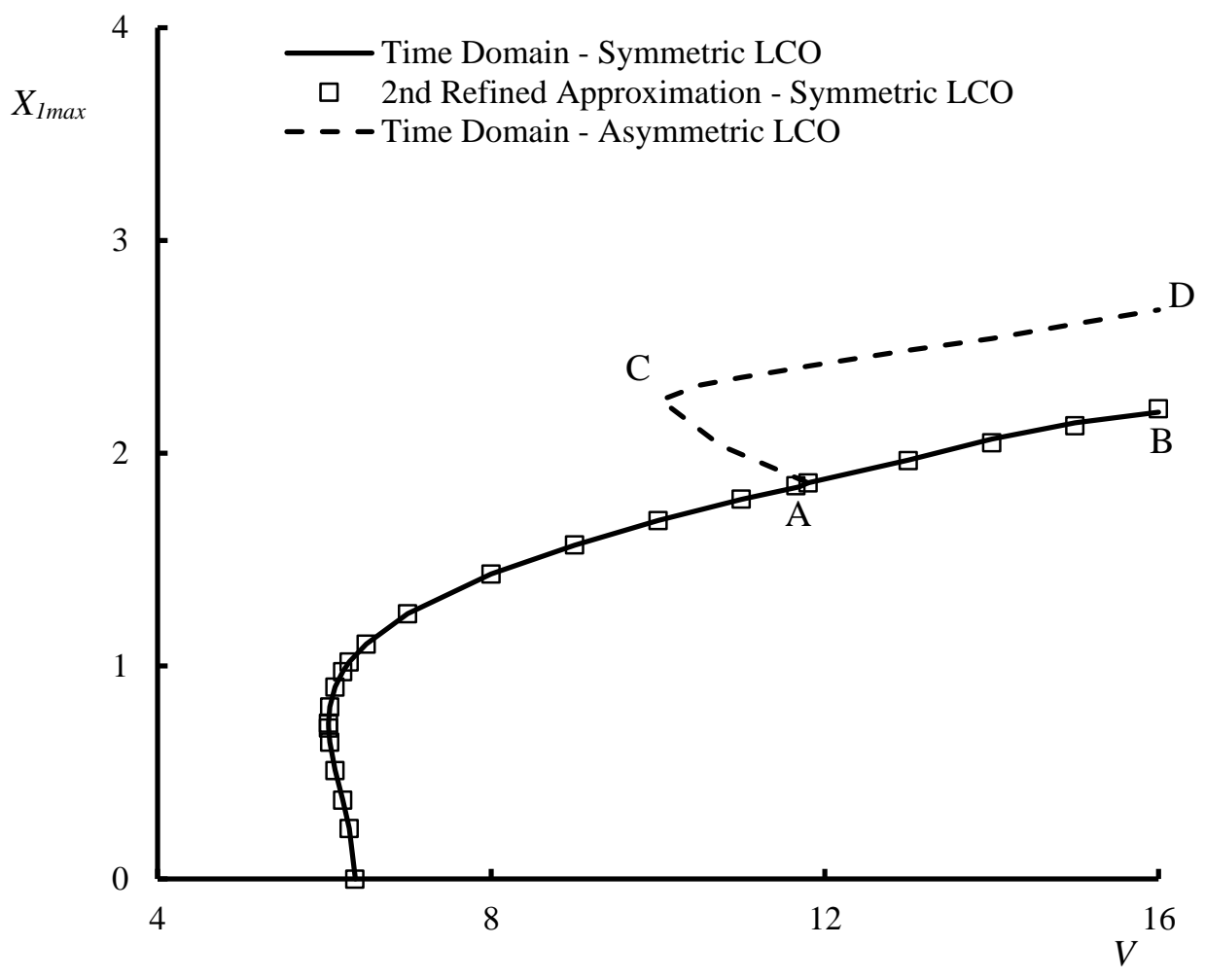

Fig. 24 Bifurcational Behaviour of Nonlinear System $-K=4.0, C=0.1$ and $\delta=2.5$ 


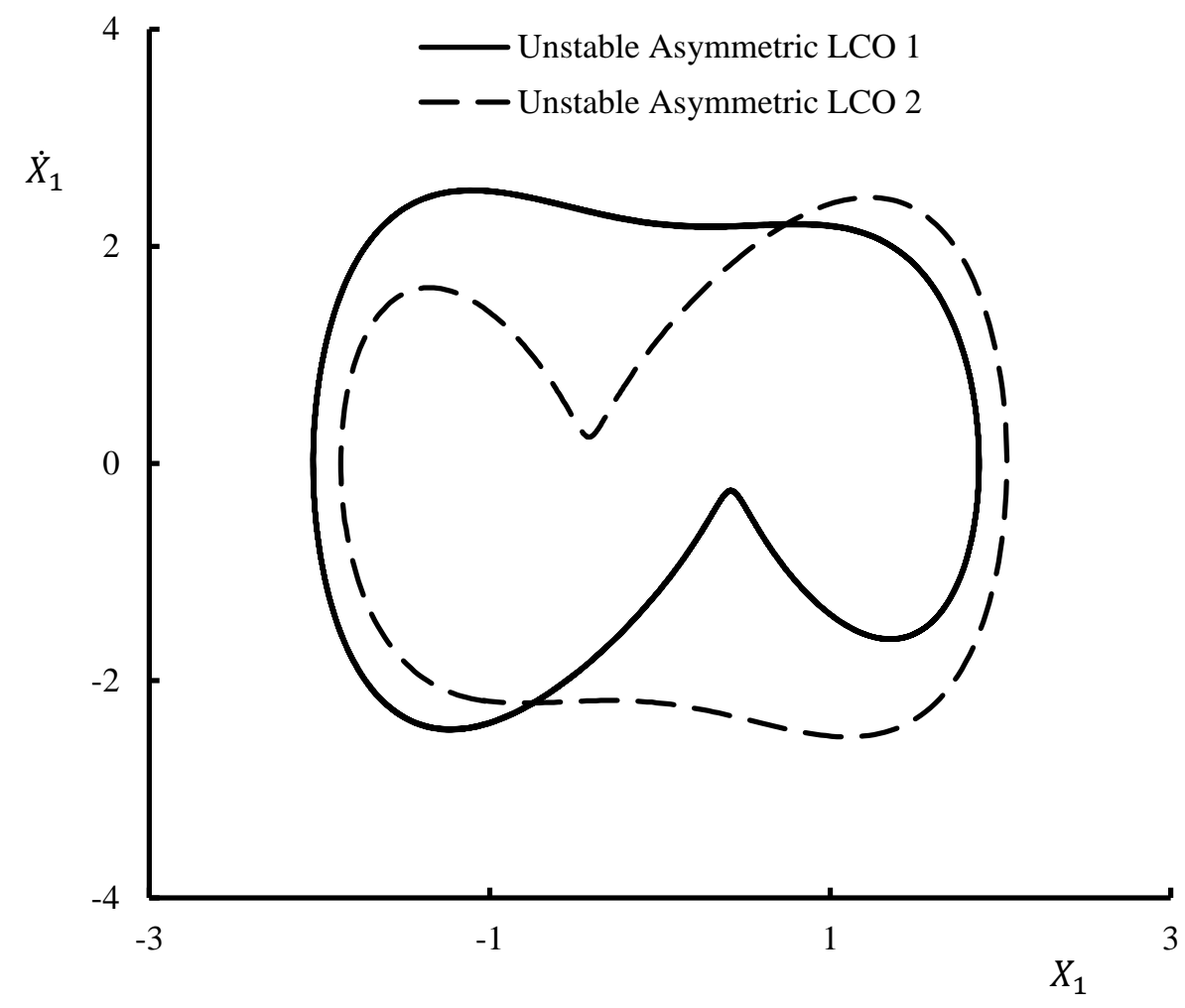

Fig. 25 Time Domain Predictions of Unstable Asymmetric Limit Cycles. $K=4.0, V=10.75, C=$ $0.10, \delta=2.5$

Further to Fig. 24, Table 2 presents results of Floquet analysis indicating the stability of the symmetric limit cycles obtained. Also, shown are predictions of the limit cycle fundamental frequency obtained from both the second refined approximation and the time domain analysis, which show very good agreement.

Table 2 Floquet Multipliers and Frequencies for Limit Cycles $-K=4.0, C=0.1, \delta=2.5$

\begin{tabular}{|c|c|c|c|c|}
\hline$V$ & Floquet Multipliers & Stability & $\begin{array}{l}\text { Frequency }-2^{\text {nd }} \\
\text { Refined } \\
\text { Approximation }\end{array}$ & $\begin{array}{c}\text { Frequency - } \\
\text { Time Domain }\end{array}$ \\
\hline 6.3 & $\begin{array}{rr}0.9258 & -0.2197 \pm 0.4290 \mathrm{i} \\
1.0251 & 0.2319 \pm 0.3847 \mathrm{i}\end{array}$ & $\begin{array}{c}\text { Stable } \\
\text { Unstable }\end{array}$ & $\begin{array}{l}1.0983 \\
1.0711\end{array}$ & $\begin{array}{l}1.0983 \\
1.0711\end{array}$ \\
\hline 7.0 & $\begin{array}{ccc}0.9266 & -0.5107 & -0.4631\end{array}$ & Stable & 1.1120 & 1.1120 \\
\hline 9.0 & $0.9312-0.2295 \pm 0.4915 \mathrm{i}$ & Stable & 1.1339 & 1.1338 \\
\hline 11.0 & $0.6469 \quad 0.3808$ & Stable & 1.1488 & 1.1485 \\
\hline 11.8 & $0.9609 \quad 0.2234$ & Unstable & 1.1538 & 1.1533 \\
\hline 14.0 & $0.9626 \quad 0.1604$ & Unstable & 1.1656 & 1.1652 \\
\hline
\end{tabular}




\section{Concluding Remarks}

Higher order approximations to limit cycles of an autonomous multi-degree-of-freedom system with a single cubic nonlinearity have been obtained by first obtaining a harmonic balance solution based on first and third harmonics which is then refined to account for the effect of higher harmonics. The method is analytical other than that a first estimate of frequency has to be obtained numerically from a polynomial equation of degree 16 . The novelty of the method lies in extending the method of [31] to a multi-degree-of-freedom system and basing it on an available harmonic balance solution containing not only first, but also third harmonics. The methodology was applied to a system derived from an aeroelastic analysis of an all-moving control surface with a nonlinearity in the torsional degree-offreedom of the root support, and parameters corresponding to air speed, together with linear stiffness and viscous damping of the root support were varied. By making comparisons with time domain simulation, the method has been shown to be successful in limit cycle prediction where higher harmonics begin to become significant with the refinement method shown to be effective. It was notable that increasing damping parameter $C$ in the system considered led to responses where the higher harmonics were significant.

A method for carrying out Floquet analysis originally developed in [34] for a first order three degreeof-freedom system and which was extended to a second order two-degree-of-freedom system in [23] has been applied to assess limit cycle stability. Given accurate limit cycle predictions, the method was shown to correctly identify limit cycle stability and where changes in stability occurred as system parameter changes occurred.

There are a number of possibilities for pursuing the approach presented in this paper further. The method for refining limit cycle approximations is based on the approach of [31] where, as noted previously, limit cycles of the Van der Pol equation with moderate values of damping coefficient were investigated. However, [32] presents a modification to the approach given in [31] which could also be applied to the type of system considered in this paper. As already noted, there has been interest in solving the equations that arise from the harmonic balance method using Groebner bases [27-29] and a further possibility would be to investigate their application, or other methods for solving nonlinear polynomial coupled equations, to obtain an 'almost analytic' solution including both even and higher harmonics. This would further refine the limit cycle predictions already obtained, enable asymmetric limit cycles to be determined and potentially reveal additional limit cycles as the picture of bifurcational behaviour of the system is extended.

The method presented in this paper is limited in that it only enables periodic solutions to be determined, with information on their stability being provided by Floquet analysis. Examples of methods which overcome this limitation and have been applied to yield transient motions leading to limit cycles in three and four-dimensional systems include the method of multiple scales [37 - 39] and second order averaging [40]. The approaches presented in those papers would be highly relevant to the system considered in this paper. 


\section{References}

1. Nayfeh, A. H., Perturbation methods. New York; Chichester; Wiley; 1973.

2. Nayfeh, A H., Introduction to Perturbation Techniques, John Wiley \& Sons, Incorporated, 1994.

3. Mickens, R. E., Truly nonlinear oscillations: Harmonic balance, parameter expansions, iteration, and averaging methods, World Scientific, ISBN 978-9814291651, 2010.

4. Dai, Honghua, Yue, Xiaokui, Yuan, Jianping, Xie, Dan, A fast harmonic balance technique for periodic oscillations of an aeroelastic airfoil, Journal of Fluids and Structures Vol 50, pages 231-252, 2014.

5. Dai, Honghua, Yue, Xiaokui, Yuan, Jianping, Xie, Dan, A time domain collocation method for studying the aeroelasticity of a two dimensional airfoil with a structural nonlinearity, Journal of Computational Physics, Vol 270, pages 214-237, 2014.

6. Dai, Honghua, Yue, Xiaokui, Yuan, Jianping, Xie, Dan, Dealiasing harmonic balance method for obtaining periodic solutions of an aeroelastic system, Aerospace Science and Technology, Vol. 77, pages 244-255, 2018.

7. Liu, L, Dowell, E. H., Hall and K. C., A novel harmonic balance analysis for the Van Der Pol oscillator, International Journal of Nonlinear Mechanics, Vol. 42, pages 2-12, 2007.

8. Detroux, T., Renson, L., Masset, L., Kerschen, G., The harmonic balance method for bifurcation analysis of large-scale nonlinear mechanical systems, Computational Methods in Applied Mechanical Engineering, Vol. 296, pages 18-38, 2015.

9. Khodaparast, H. H., Madinei, H., Friswell, M. I., Adhikari, S., Coggon, S., Cooper, J. E., An extended harmonic balance method based on incremental nonlinear control parameters, Mechanical Systems and Signal Processing, Vol. 85, pages 716-729, 2017.

10. Liping, L., Dowell, E. H., Thomas, J. P., Higher Order Harmonic Balance Analysis for Limit Cycle Oscillations in an Airfoil with Cubic Restoring Forces, AIAA 2005-1918, 46th

AIAA/ASME/ASCE/AHS/ASC Structures, Structural Dynamics \& Materials Conference, 18 - 21 April 2005, Austin, Texas.

11. Liu, L., Dowell, E. H., The Secondary Bifurcation of an Aeroelastic Airfoil Motion: Effect of High Harmonics, Nonlinear Dynamics, Vol. 37, pages 31-49, 2004.

12. Padmanabhan, M.A., Dowell, E.H., Thomas, J.P., Pasiliao, C.L., Store-induced limit-cycle oscillations due to nonlinear wing-store attachment, Journal of Aircraft, Vol. 53 (3), pages. 778789, 2016.

13. Liu, L., Dowell, E.H., High dimensional harmonic balance analysis for dynamic piecewise aeroelastic systems, ASME International Mechanical Engineering Congress and Exposition, Proceedings, 12, pages 659-669, 2009.

14. Liu, L., Dowell, E.H., Thomas, J.P., A high dimensional harmonic balance approach for an aeroelastic airfoil with cubic restoring forces, Journal of Fluids and Structures, 23(3), pages 351-363, 2007.

15. Liu, L., Dowell, E.H., Thomas, J.P., Higher order harmonic balance analysis for limit cycle oscillations in an airfoil with cubic restoring forces, Collection of Technical Papers -

AIAA/ASME/ASCE/AHS/ASC Structures, Structural Dynamics and Materials Conference, 3, pages 1467-1476. 2005.

16. Vio, G. A., Dimitriadis, G., Cooper, J. E., Improved implementation of the harmonic balance method, 48th AIAA/ASME/ASCE/AHS/ASC Structures, Structural Dynamics \& Materials Conference, Vol. 8, page 7879-7890, 23 - 26 April 2007, Waikiki, Hawaii.

17. Chan, H. S. Y., Chung, K. W., and Xu, Z., A Perturbation-Iterative Method for Determining the Limit Cycles of Strongly Non-Linear Oscillators, Journal of Sound and Vibration, Vol. 183, No. 4, pages 707-717, 1995. 
18. Chan, H. S. Y., Chung, K. W., and Xu, Z., A Perturbation-Incremental Method for Strongly Non-Linear Oscillators, International Journal of Nonlinear Mechanics, Vol. 31, No. 1, pages 59-72, 1996.

19. Chung K. W., Chan C. L., Xu Z., Mahmoud G. M., A Perturbation-Incremental Method for Strongly Nonlinear Autonomous Oscillators with Many Degrees of Freedom. Nonlinear Dynamics, Vol. 28(3), pages 243-59, 2002.

20. Chung, K. W., Chan, C. L., Lee, B. H. K., Bifurcation analysis of a two-degree-of-freedom aeroelastic system with freeplay structural nonlinearity by a perturbation-incremental method, Journal of Sound and Vibration, Vol. 299, pages 520-539, 2007.

21. Xuechuan W, Xiaokui Y, Honghua D, Jianping Y, Analysis of a Two-Dimensional Aeroelastic system Using the Differential Transform Method, Journal of Computational nonlinear dynamics, 2016.

22. Qian, Y. H. and Zhang, Y. F., Optimal extended homotopy analysis for Multi-Degree-ofFreedom nonlinear dynamical systems and its application, Structural Engineering and Mechanics, Vol. 61, No. 1, pages 105-116, 2017.

23. Lewis, A. P., Refined Analytical Approximations to Limit Cycles for Non-Linear MultiDegree-of-Freedom Systems, International Journal of Non-linear Mechanics, Vol. 110, pages 58-68 (2019).

24. Kovacic, I., Cveticanin, L., Zukovic, M., Rakaric, R., Jacobi elliptic functions: A review of nonlinear oscillatory application problems, Journal of Sound and Vibration, Vol. 380, pages 1-36, 2016.

25. Chen, Y. M., Liu, J. K., Elliptic harmonic balance method for two degree-of-freedom selfexcited oscillators, Communications in Nonlinear Science and Numerical Simulation, Vol. 14, pages 916-922, 2009.

26. Niu, Y. B., Wang, Z. W., Flutter analysis of 2-D airfoil with nonlinearities using elliptic harmonic balance method, Gongcheng Lixue/Engineering Mechanics, Vol. 30, No. 4, pages 461-465, 2013.

27. Liu, J., Peddieson, J., Evaluation of Groebner Basis methodology as an Aid to Harmonic Balance, Journal of vibration and acoustics, Vol. 136, pages 024502-1 - 024502-4, 2013.

28. Liu, J., Peddieson, J., Application of Groebner Basis Methodology to Nonlinear Mechanics problems, Hong H., Yap C. (eds) Mathematical Software - ICMS 2014. ICMS 2014. Lecture Notes in Computer Science, Vol 8592. Springer, Berlin, Heidelberg, 2014.

29. Grolet, A., Thouverez, F., Computing multiple periodic solutions of nonlinear vibration problems using the harmonic balance method and Groebner bases, Mechanical Systems and Signal processing, Volume 52-53, pages 529-547, 2015.

30. Lee, B. H. K., Liu, L, Chung, K. W., Airfoil motion in subsonic flow with strong cubic nonlinear restoring forces, Journal of Sound and Vibration, Vol. 281, pages 699-717, 2005.

31. Qui, Shui-Sheng, Filanovsky, I. M., Periodic Solutions of the Van der Pol Equation with Moderate Values of Damping Coefficient, IEEE Transactions on Circuits and Systems, Vol. CAS-34, No. 8, August 1987.

32. Qui, Shui-Sheng, Filanovsky, A Method of Calculation of Steady state Oscillations in Autonomous Non-Linear systems, Journal of Sound and Vibration, Vol. 136, No. 1, pages 3544 (1990).

33. Popov, E. P., On the use of the Harmonic Linearization Method in Automatic Control theory, NACA TM 1406, 1957.

34. Bonani, F. and Gilli, M, Analysis of Stability and Bifurcations of Limit Cycles in Chua's Circuit Through the Harmonic Balance Approach, IEEE Transactions on Circuits and Systems - I: Fundamental Theory and Applications, Vol. 46, No. 8, August 1999.

35. Ashley, H., Zartarian, G., Piston Theory - a New Aerodynamic Tool for the Aeroelastician, Journal of the Aeronautical Sciences, Vol. 23, pages 1109-1118, 1956. 
36. Itovich, G. R., Moiola J. L., On period doubling bifurcations of cycles and the harmonic balance method, Chaos, Solitons and Fractals, Vol. 27, pages 647 - 665, 2006.

37. Morino, L., A Perturbation Method for Treating Nonlinear Panel Flutter problems, AIAA Journal, Vol. 7, No. 3, pages 405 - 411, 1969.

38. Nayfeh, A. H., Balachandran, B., Motion Near a Hopf Bifurcation of a Three-Dimensional System, Mechanics Research Communications, Vol. 17, No. 4, pages 191-198, 1990.

39. Balachandran, B., Nayfeh, A. H., Cyclic Motion Near a Hopf Bifurcation of a FourDimensional System, Nonlinear Dynamics, Vol. 1, 1992, pages 19-39, 1992.

40. Banerjee, B., Bajaj, A. K., Davies, P., Resonant Dynamics of an Autoparametric System: A Study Using Higher Order Averaging, International Journal of Nonlinear Mechanics, Vol. 31, No. 1, pages 21-39, 1996.

\section{APPENDIX A Expressions for Coefficients in the Harmonic Balance Equations of Section 2}

The coefficients of $a_{1}, a_{3}, b_{3}, c_{1}, d_{1}, c_{3}, d_{3}$ introduced in Equations (4) to (7) are functions of the coefficients of $G_{\mathrm{ij}}$ and $H_{i j}$ in Equation (1) and are given by:

$$
\begin{gathered}
m_{1}=H_{21} ; p_{1}=H_{22}-\omega^{2} ; q_{1}=-\omega G_{22} \\
m_{2}=\omega G_{21} ; p_{2}=-q_{1}=\omega G_{22} ; q_{2}=p_{1}=H_{22}-\omega^{2} \\
m_{3}=\omega G_{11} ; p_{3}=\omega G_{12} ; q_{3}=H_{12} \\
E_{1}=-\frac{\left(m_{1} p_{1}+m_{2} p_{2}\right)}{\left(p_{1}^{2}+p_{2}^{2}\right)} ; F_{1}=\frac{\left(m_{1} p_{2}-m_{2} p_{1}\right)}{\left(p_{1}^{2}+p_{2}^{2}\right)} \\
M_{3}=m_{3}+p_{3} E_{1}+q_{3} F_{1} ; M_{4}=m_{4}+p_{4} E_{1}+q_{4} F_{1} \\
m_{23}=n_{13}=H_{21} ; n_{23}=-m_{13}=-3 \omega G_{21} ; n_{13}=H_{21} ; p_{23}=q_{13}=H_{22}-9 \omega^{2} \\
m_{4}=H_{11}-\omega^{2} ; p_{4}=H_{12}=q_{3} ; q_{4}=-\omega G_{12}=-p_{3} \\
m_{13}=3 \omega G_{21} ; n_{13}=H_{21} ; p_{13}=3 \omega G_{22} ; q_{13}=H_{22}-9 \omega^{2}
\end{gathered}
$$




$$
\begin{gathered}
m_{33}=3 \omega G_{11} ; n_{33}=H_{11}-9 \omega^{2} ; p_{33}=-q_{43}=3 \omega G_{12} ; q_{33}=H_{12} \\
m_{43}=n_{33}=H_{11}-9 \omega^{2} ; n_{43}=-m_{33}=-3 \omega G_{11} ; p_{43}=q_{33}=H_{12} \\
E_{3}=-\frac{\left(m_{13} p_{13}+m_{23} p_{23}\right)}{\left(p_{13}^{2}+p_{23}^{2}\right)} ; \quad F_{3}=\frac{\left(m_{13} p_{23}-m_{23} p_{13}\right)}{\left(p_{13}^{2}+p_{23}^{2}\right)} \\
M_{33}=-N_{43}=m_{33}+p_{33} E_{3}-q_{33} F_{3} ; N_{33}=n_{33}+p_{33} F_{3}+q_{33} E_{3} \\
M_{43}=N_{33}=m_{43}+p_{43} E_{3}-q_{43} F_{3} ; N_{43}=n_{43}+p_{43} F_{3}+q_{43} E_{3}
\end{gathered}
$$

APPENDIX B Expressions for $\mathbf{C}_{10}, C_{30}, C_{50}, C_{70}, C_{90}, S_{10}, S_{30}, S_{50}, S_{70}, S_{90}$ in Section 3

$$
\begin{gathered}
C_{10}=-\frac{3}{4} a_{10}^{2} b_{30} \\
S_{10}=\frac{3}{4}\left(a_{10}^{3}-a_{10}^{2} a_{30}+2 a_{10}\left(a_{30}^{2}+b_{30}^{2}\right)\right) \\
C_{30}=\frac{3}{4}\left(2 a_{10}^{2} b_{30}+b_{30}\left(a_{30}^{2}+b_{30}^{2}\right)\right) \\
S_{30}=\frac{1}{4}\left(-a_{10}^{3}+6 a_{10}^{2} a_{30}+3 a_{30}\left(a_{30}^{2}+b_{30}^{2}\right)\right) \\
C_{50}=-\frac{1}{4}\left(3 b_{30} a_{10}^{2}-6 a_{30} b_{30} a_{10}\right) \\
S_{50}=-\frac{1}{4}\left(3 a_{10}^{2} a_{30}-3 a_{10} a_{30}^{2}+3 a_{10} b_{30}^{2}\right)
\end{gathered}
$$




$$
\begin{gathered}
C_{70}=-\frac{3}{2} a_{10} a_{30} b_{30} \\
S_{70}=-\frac{1}{4}\left(3 a_{10} a_{30}^{2}-3 a_{10} b_{30}^{2}\right) \\
C_{90}=-\frac{1}{4}\left(3 a_{30}^{2} b_{30}-b_{30}^{3}\right) \\
S_{90}=\frac{1}{4}\left(-a_{30}^{3}+3 a_{30} b_{30}^{2}\right)
\end{gathered}
$$

\section{APPENDIX C Expressions for $\mathbf{C}_{11}, C_{31}, C_{51}, C_{71}, C_{91}, S_{11}, S_{31}, S_{51}, S_{71}, S_{91}$ in Section 3}

$$
\begin{aligned}
C_{11}=\frac{1}{4} b_{30}^{2} b_{51} & -\frac{1}{4} a_{30}^{2} b_{71}-\frac{1}{4} a_{30}^{2} b_{51}+\frac{1}{4} b_{30}^{2} b_{71}-\frac{1}{2} a_{10} a_{30} b_{51}+\frac{1}{2} a_{10} a_{51} b_{30} \\
& +\frac{1}{2} a_{30} a_{51} b_{30}+\frac{1}{2} a_{30} a_{71} b_{30} \\
S_{11}=\frac{1}{4} a_{30}^{2} a_{51} & -\frac{1}{4} a_{30}^{2} a_{71}-\frac{1}{4} b_{30}^{2} a_{51}+\frac{1}{4} b_{30}^{2} a_{71}-\frac{1}{2} a_{10} a_{30} a_{51}-\frac{1}{2} a_{10} b_{51} b_{30} \\
& +\frac{1}{2} a_{30} b_{51} b_{30}-\frac{1}{2} a_{30} b_{71} b_{30} \\
C_{31}=\frac{1}{4} b_{30}^{2} b_{91}- & \frac{1}{4} a_{30}^{2} b_{91}-\frac{1}{4} a_{10}^{2} b_{51}+\frac{1}{2} a_{10} a_{30} b_{51}-\frac{1}{2} a_{10} a_{51} b_{30}-\frac{1}{2} a_{10} a_{30} b_{71} \\
+ & \frac{1}{2} a_{10} a_{71} b_{30}+\frac{1}{2} a_{30} a_{91} b_{30} \\
& +\frac{1}{2} a_{30}^{2} a_{91}-\frac{1}{4} a_{10}^{2} a_{51}+\frac{1}{2} a_{10} a_{51} a_{30}-\frac{1}{2} a_{10} a_{30} a_{71}+\frac{1}{2} a_{10} b_{51} b_{30} a_{30} b_{71}-\frac{1}{2} a_{10} a_{91} b_{30} \\
& -\frac{1}{2} a_{10} b_{71} b_{30}-\frac{1}{2} a_{30} b_{91} b_{30} \\
S_{31}=\frac{1}{4} b_{30}^{2} a_{91} & -\frac{1}{2} a_{10} a_{30} b_{91}+\frac{1}{2} a_{10} a_{91} b_{30} \\
S_{51}=\frac{1}{2} a_{10}^{2} a_{51} & -\frac{1}{4} a_{10}^{2} a_{71}+\frac{1}{2} a_{30}^{2} a_{51}+\frac{1}{2} b_{30}^{2} a_{51}+\frac{1}{2} a_{10} a_{30} a_{71}-\frac{1}{2} a_{10} a_{91} a_{30} \\
C_{51}=\frac{1}{2} a_{10}^{2} b_{51} & -\frac{1}{4} a_{10}^{2} b_{71}+\frac{1}{2} a_{30}^{2} b_{51}+\frac{1}{2} b_{30}^{2} a_{51}+\frac{1}{2} a_{10} a_{30} b_{71}-\frac{1}{2} a_{10} a_{71} b_{30} \\
& \\
& \\
& \\
&
\end{aligned}
$$




$$
\begin{aligned}
C_{71}=\frac{1}{2} a_{10}^{2} b_{71} & -\frac{1}{4} a_{10}^{2} b_{51}-\frac{1}{4} a_{10}^{2} b_{91}+\frac{1}{2} a_{30}^{2} b_{71}+\frac{1}{2} b_{30}^{2} b_{71}+\frac{1}{2} a_{10} a_{30} b_{51} \\
+ & \frac{1}{2} a_{10} a_{51} b_{30}+\frac{1}{2} a_{10} a_{30} b_{91}-\frac{1}{2} a_{10} a_{91} b_{30} \\
S_{71}=\frac{1}{2} a_{10}^{2} a_{71} & -\frac{1}{4} a_{10}^{2} a_{51}-\frac{1}{2} a_{10}^{2} a_{91}+\frac{1}{2} a_{30}^{2} a_{71}+\frac{1}{2} b_{30}^{2} a_{71}+\frac{1}{2} a_{10} a_{30} a_{51} \\
& +\frac{1}{2} a_{10} a_{91} a_{30}-\frac{1}{2} a_{10} a_{30} b_{51}+\frac{1}{2} a_{10} a_{91} b_{30} \\
C_{91}=\frac{1}{2} a_{10}^{2} b_{91}- & \frac{1}{4} a_{10}^{2} b_{71}+\frac{1}{2} a_{30}^{2} b_{91}+\frac{1}{2} b_{30}^{2} b_{91}-\frac{1}{2} a_{10} a_{30} b_{51}-\frac{1}{2} a_{10} a_{51} b_{30} \\
+ & \frac{1}{2} a_{10} b_{71} a_{30}+\frac{1}{2} a_{10} a_{71} b_{30} \\
S_{91}=\frac{1}{2} a_{10}^{2} a_{91}- & \frac{1}{4} a_{10}^{2} a_{71}+\frac{1}{2} a_{30}^{2} a_{91}+\frac{1}{2} b_{30}^{2} a_{91}-\frac{1}{2} a_{10} a_{30} a_{51}+\frac{1}{2} a_{10} a_{71} a_{30} \\
+ & \frac{1}{2} a_{10} b_{30} b_{51}-\frac{1}{2} a_{10} a_{71} b_{30}
\end{aligned}
$$

